\title{
New Theories on Boundary Layer Transition and Turbulence Formation
}

\author{
Chaoqun Liu, Ping Lu, Lin Chen, and Yonghua Yan \\ Department of Mathematics, The University of Texas at Arlington, Arlington, TX 76019, USA \\ Correspondence should be addressed to Chaoqun Liu, cliu@uta.edu
}

Received 26 January 2012; Accepted 25 March 2012

Academic Editor: Guan Heng Yeoh

Copyright () 2012 Chaoqun Liu et al. This is an open access article distributed under the Creative Commons Attribution License, which permits unrestricted use, distribution, and reproduction in any medium, provided the original work is properly cited.

This paper is a short review of our recent DNS work on physics of late boundary layer transition and turbulence. Based on our DNS observation, we propose a new theory on boundary layer transition, which has five steps, that is, receptivity, linear instability, large vortex structure formation, small length scale generation, loss of symmetry and randomization to turbulence. For turbulence generation and sustenance, the classical theory, described with Richardson's energy cascade and Kolmogorov length scale, is not observed by our DNS. We proposed a new theory on turbulence generation that all small length scales are generated by "shear layer instability" through multiple level ejections and sweeps and consequent multiple level positive and negative spikes, but not by "vortex breakdown." We believe "shear layer instability" is the "mother of turbulence." The energy transferring from large vortices to small vortices is carried out by multiple level sweeps, but does not follow Kolmogorov's theory that large vortices pass energy to small ones through vortex stretch and breakdown. The loss of symmetry starts from the second level ring cycle in the middle of the flow field and spreads to the bottom of the boundary layer and then the whole flow field.

\section{Introduction}

These comments clearly show that the mechanism of turbulence formation and sustenance is still a mystery for research. Note that both Heisenberg and Lamb were not optimistic for the turbulence study.

1.1. Turbulence Is a Mystery. Turbulence is still covered by a mystical veil in nature after over a century of intensive study. Following comments are made by Wikipedia web page at http://en.wikipedia.org/wiki/Turbulence "Nobel Laureate Richard Feynman described turbulence as "the most important unsolved problem of classical physics" (USA Today 2006). According to an apocryphal story, Werner Heisenberg (another Nobel Prize winner) was asked what he would ask God, given the opportunity. His reply was "When I meet God, I am going to ask him two questions: Why relativity? And why turbulence? I really believe he will have an answer for the first." [1]. Horace Lamb was quoted as saying in a speech to the British Association for the Advancement of Science, "I am an old man now, and when I die and go to heaven there are two matters on which I hope for enlightenment. One is quantum electrodynamics, and the other is the turbulent motion of fluids. And about the former I am rather optimistic" $[2,3]$.

1.2. Richardson's Vortex and Energy Cascade Theory (1928). Richardson believed that a turbulent flow is composed by "eddies" of different sizes. The large eddies will be stretching, unstable, and breaking up to smaller eddies. These smaller eddies undergo the same process, giving rise to even smaller eddies. This process will continue until reaching a sufficiently small length scale such that the viscosity of the fluid can effectively dissipate the kinetic energy into internal energy. During the process of vortex breakdown, the kinetic energy of the initial large eddy is divided into the smaller eddies.

1.3. Kolmogorov Assumption (1941). The classical theory on turbulence was given by Kolmogorov, a famous Russian mathematician. In general, the large scales of a flow are not isotropic, because they are determined by the particular boundary conditions. Agreeing with Richardson, Kolmogorov assumed that in Richardson's energy cascade, this geometrical and directional information is lost while the 


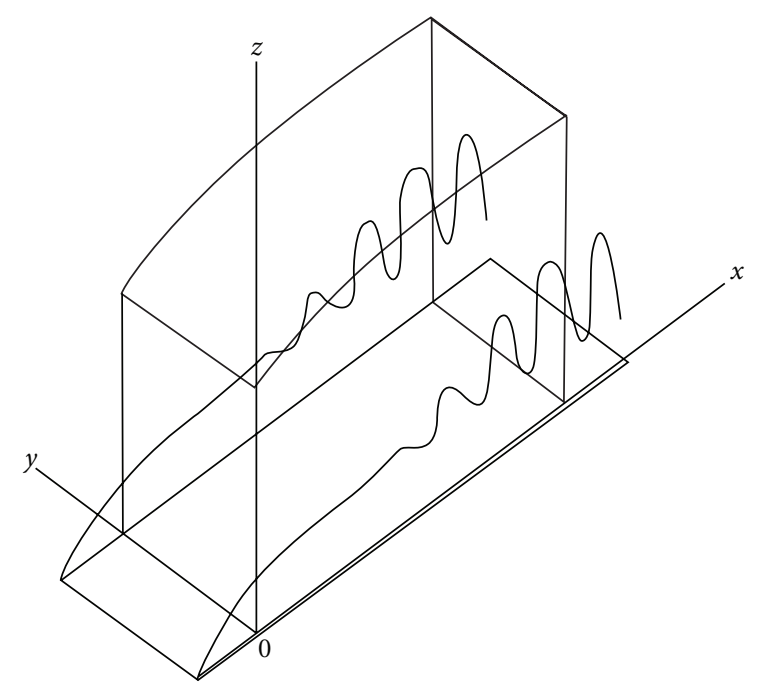

FIgURE 1: Computation domain.

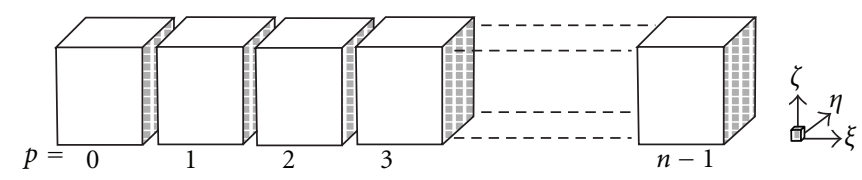

FIGURE 2: Domain decomposition along the streamwise direction in the computational space.

scale is reduced so that the statistics of the small scales have a universal character-they are statistically isotropic for all turbulent flows when the Reynolds number is sufficiently high. It was assumed that there is no dissipation during the energy transfer from large vortex to small vortex through "vortex breakdown."

1.4. Kolmogorvo's First and Second Hypotheses (1941). Based on his assumption, Kolmogorov [6,7] further gave very famous theories on the smallest length scale, which is later called Kolmogorov scale (first hypothesis), and turbulence energy spectrum (second hypothesis):

$$
\begin{aligned}
& \eta=\left(\frac{\nu^{3}}{\varepsilon}\right)^{1 / 4} \\
& E(k)=C \varepsilon^{2 / 3} k^{-5 / 3},
\end{aligned}
$$





where, $\eta$ is Kolmogorov scale, $v$ is kinematic viscosity, $\varepsilon$ is the rate of turbulence dissipation, $E$ is the energy spectrum function, $C$ is a constant and $\kappa$ is the wave number. These formulas were obtained by Kolmogorov's hypotheses that the small length scales are determined by $v$ and $\varepsilon$, and $E$ is related to $\kappa$ and $\varepsilon$. These formulas are unique according to the dimensional analysis [8].

If all small vortices are generated by "shear layer instability" but not "vortex breakdown", Kolmogorov's first hypothesis will lose the background. The smallest length scales will be related to stability of the weakest unstable "shear layer". This will require a deep study of "shear layer instability" not only "dimensional analysis".

1.5. A Short Review of Study on Late Boundary Layer Transition. The transition process from laminar to turbulent flow in boundary layers is a basic scientific problem in modern fluid mechanics. After over a hundred years of study on flow transition, the linear and weakly non-linear stages of flow transition are pretty well understood $[10,11]$. However, for late non-linear transition stages, there are still many questions remaining for research [4, 12-16]. Adrian [17] described hairpin vortex organization in wall turbulence, but did not discuss the sweep and ejection events and the role of the shear layer instability. Wu and Moin [18] reported a new DNS for flow transition on a flat plate. They did obtain fully developed turbulent flow with structure of forest of ring-like vortices by flow transition at zero pressure gradients. However, they did not give the mechanism of the late flow transition. The important mechanism of boundary layer transition such as sweeps, ejections, and positive spikes cannot be found from that paper. Recently, Guo et al. [19] conducted an experimental study for late boundary layer 


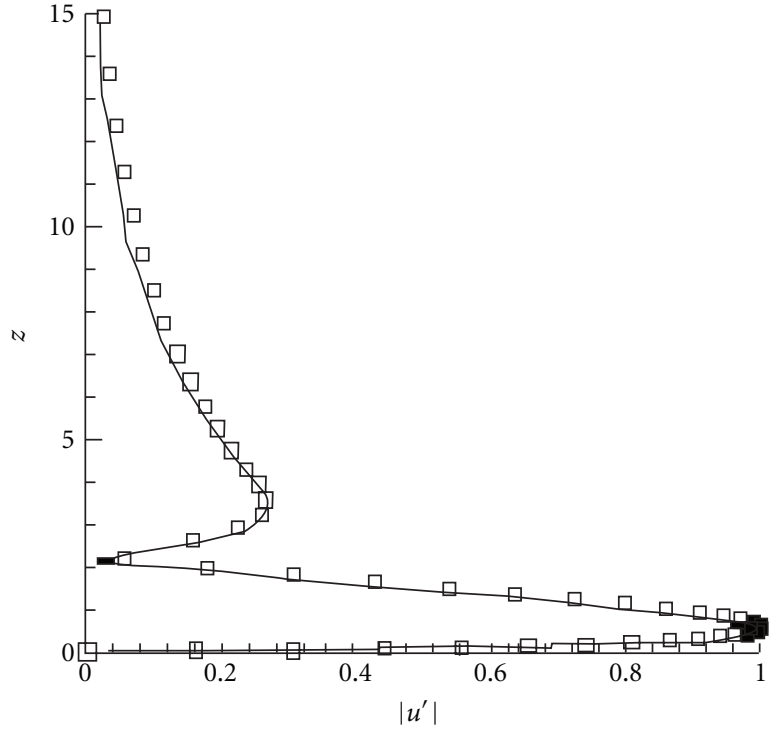

- Numerical result

$\square \quad$ LST result

(a)

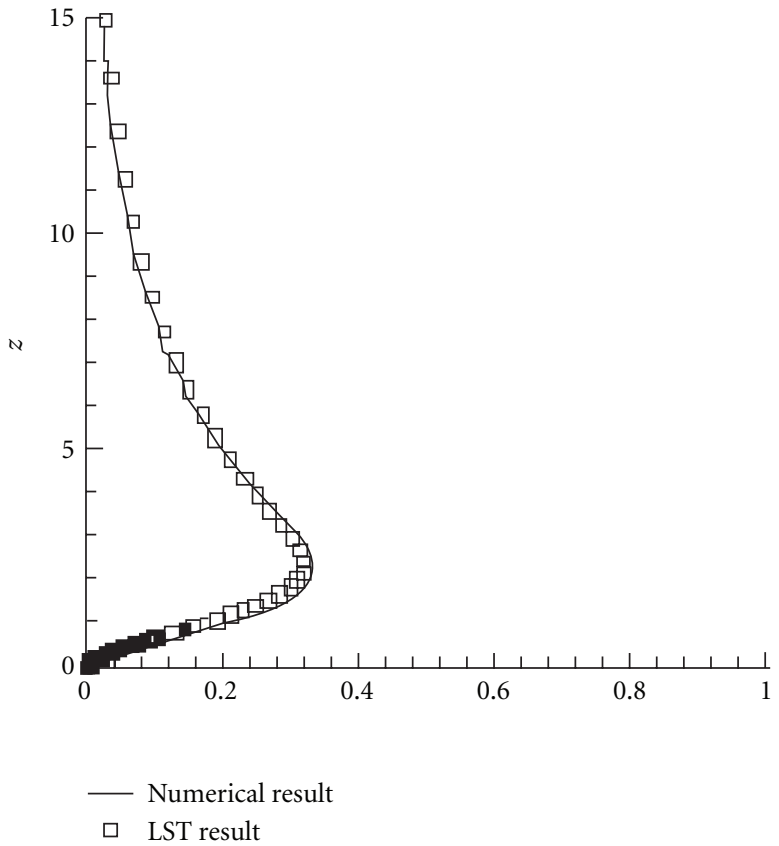

(b)

FIGURE 3: Comparison of the numerical and LST velocity profiles at Rex $=394300$.

transition in more details. They concluded that the U-shaped vortex is a barrel-shaped head wave, secondary vortex, and is induced by second sweeps and positive spikes.

In order to get deep understanding the mechanism of the late flow transition in a boundary layer and physics of turbulence, we recently conducted a high order direct numerical simulation (DNS) with $1920 \times 241 \times 128$ gird points and about 600,000 time steps to study the mechanism of the

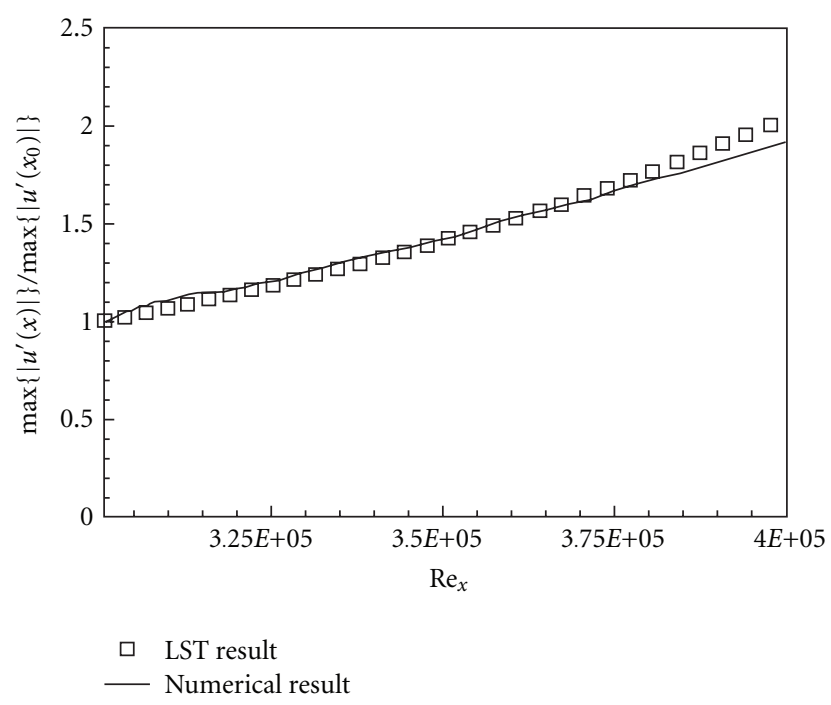

Figure 4: Comparison of the perturbation amplification rate between DNS and LST.

late stages of flow transition in a boundary layer at a free stream Mach number 0.5 [20-32]. The work was supported by AFOSR, UTA, TACC and NSF Teragrid. A number of new observations are made and new mechanisms are revealed in late boundary layer transition (LBLT) including:

(i) mechanism on secondary and tertiary vortex formation;

(ii) mechanism on first ring-like vortex formation;

(iii) mechanism on second sweep formation;

(iv) mechanism on high share layer formation;

(v) mechanism on positive spike formation;

(vi) mechanism on multiple ring formation;

(vii) mechanism on U-shaped vortex formation;

(viii) mechanism on small length vortices generation;

(ix) mechanism on multiple level high shear layer formation;

(x) mechanism of energy transfer paths;

(xi) mechanism on symmetry loss or so called "flow randomization;"

(xii) mechanism on thickening of turbulence boundary layer;

(xiii) mechanism of high surface friction of turbulent flow.

A $\lambda_{2}$ technology developed by Jeong and Hussain [33] is used for visualization.

1.6. Different Physics Observed by Our High-Order DNS. According to our recent DNS, "vortex breakdown" is not observed $[24,29,30]$. Liu gave a new theory that "turbulence is not generated by vortex breakdown but shear layer instability" [24, 30,32]. Liu believes that "shear layer instability" is the "mother of turbulence." 


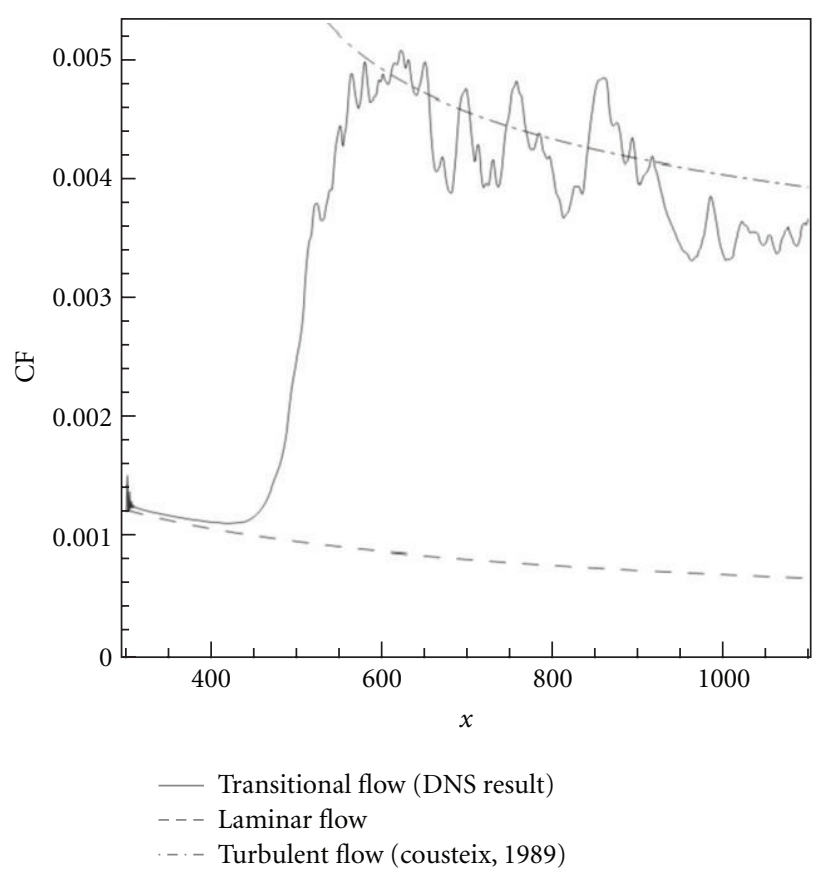

(a) Coarse Grids $(960 \times 64 \times 121)$



(b) Fine Grids $(1920 \times 128 \times 241)$

FIGURE 5: Streamwise evolutions of the time- and spanwise-averaged skin-friction coefficient.

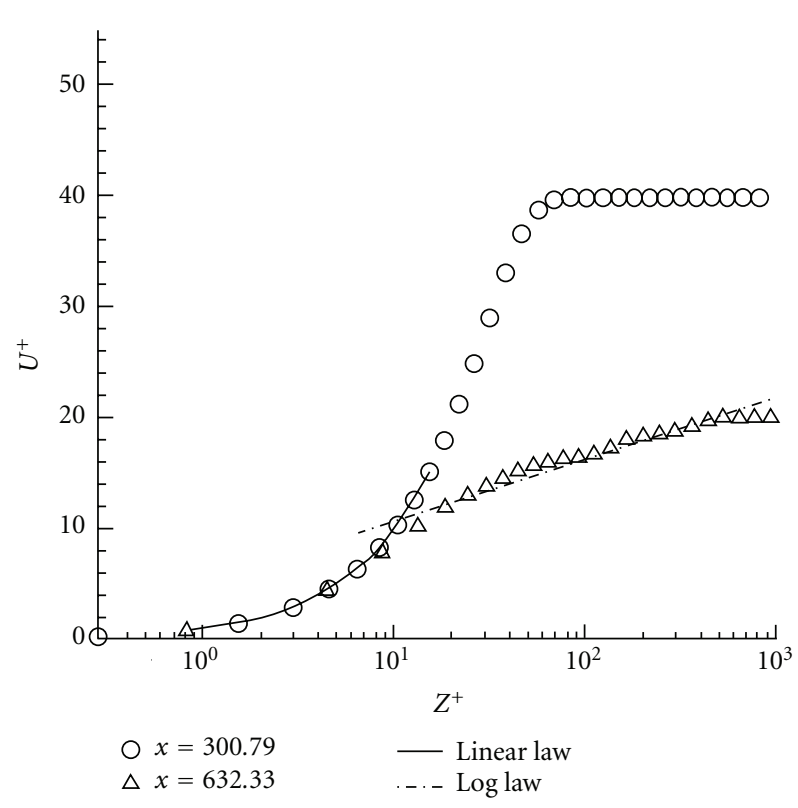

(a) Coarse Grids $(960 \times 64 \times 121)$

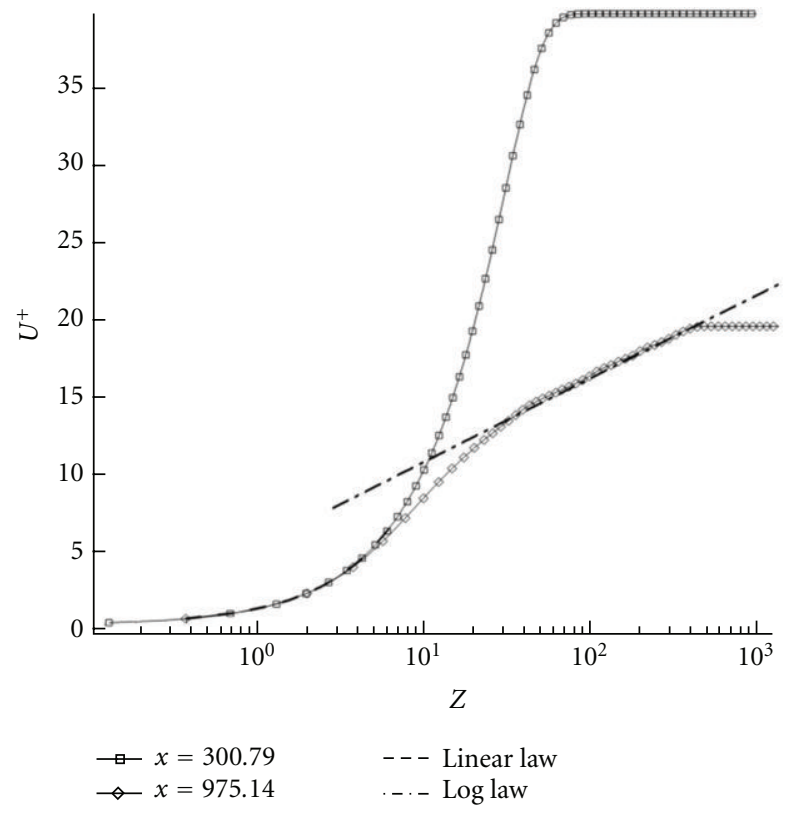

(b) Fine Grids $(1920 \times 128 \times 241)$

FIGURE 6: Log-linear plots of the time- and spanwise-averaged velocity profile in wall unit.

As Richardson's energy cascade and Kolmogorov's assumption about "vortex breakdown" are challenged, Kolmogorov's assumption that the smallest vortices are statistically isotropic becomes questionable. Since the smallest vortices are generated by the "shear layer instability," which is closely related to the shape of body configuration, they cannot be isotropic.
1.7. Questions to Classical Theory on Boundary Layer Transition. The classical theory, which considers "vortex breakdown" as the last stage of boundary layer transition on a flat plate, is challenged and the phenomenon of "hairpin vortex breakdown to smaller structures" is not observed by our new DNS [24, 27, 28]. The so-called "spikes" are actually a process of multibridge or multiring formation, which is 


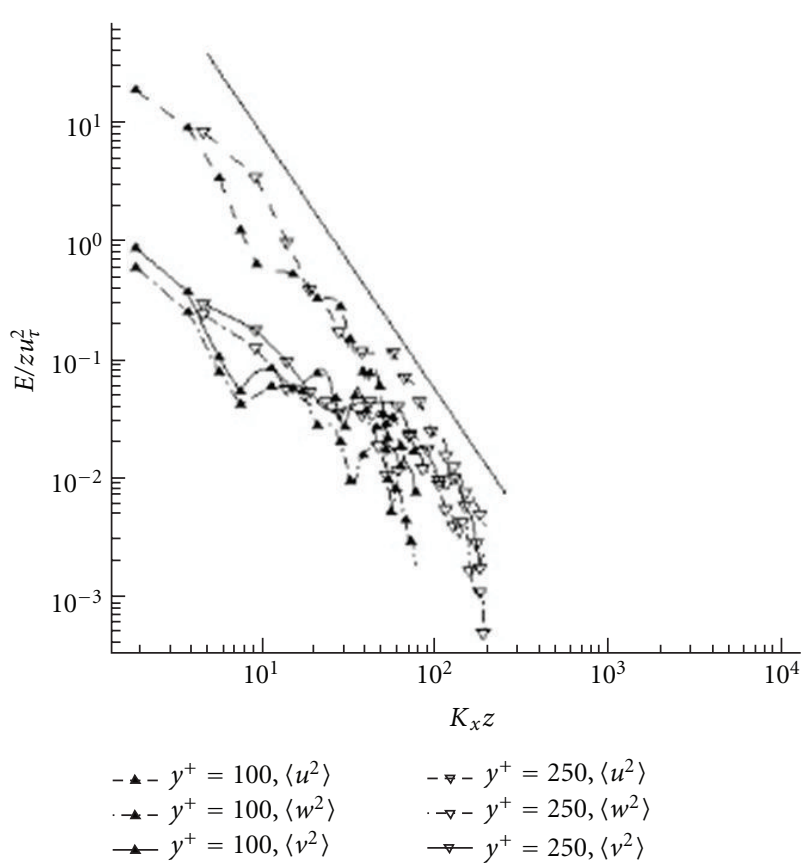

(a)

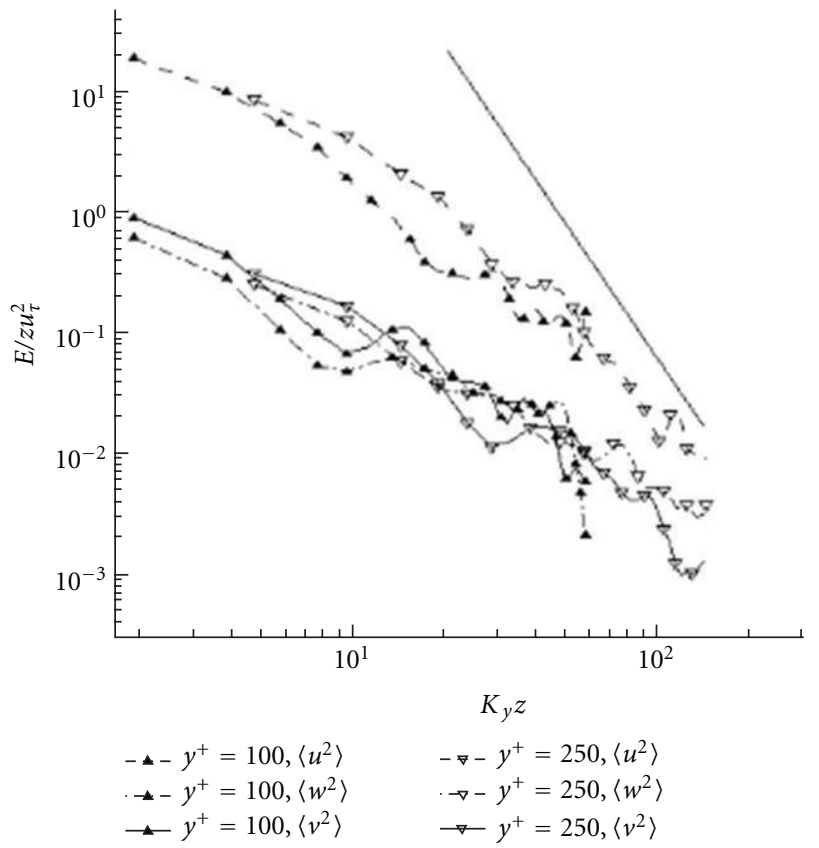

(b)

FIgURE 7: (a) Spectra in $x$-direction (b) Spectra in $y$-direction.

a rather stable large vortex structure and can travel for a long distance.

As experiment is quite expensive and has very limited power in date acquisition, direct numerical simulation (DNS) becomes a more and more important tool to discover physics. The purpose of this work is to find physics of turbulence by direct numerical simulation. The paper is

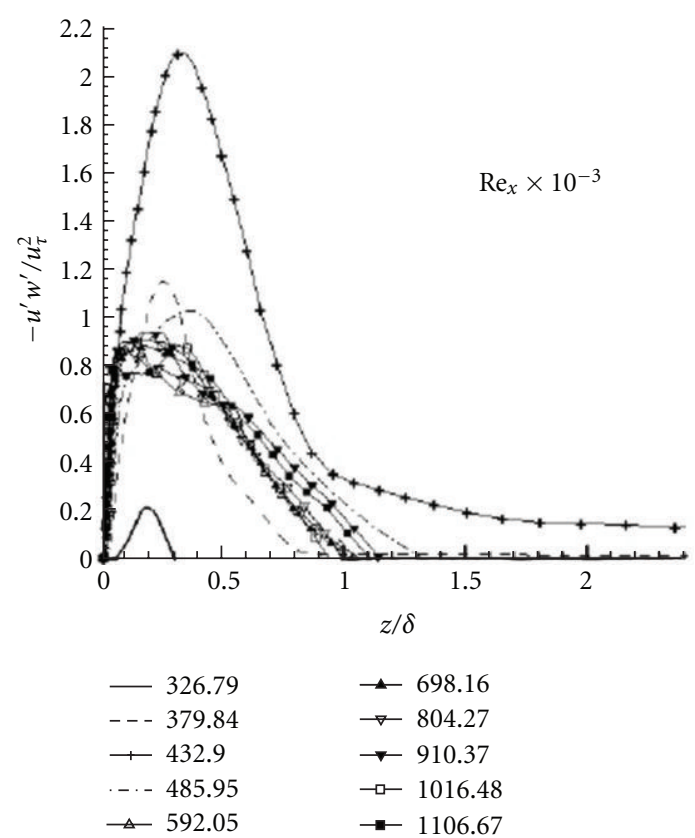

Figure 8: Reynolds stress.



(a) Our DNS

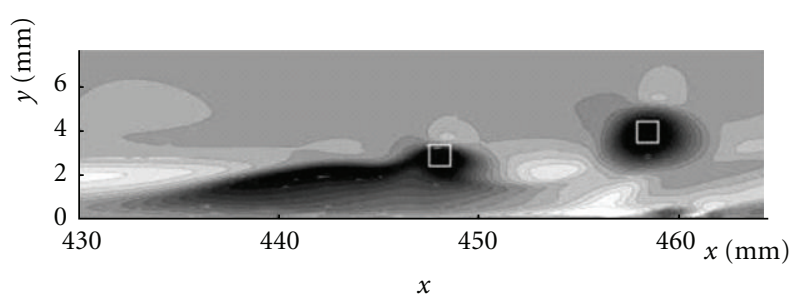

(b) Borodulin et al. [4]

FIgURE 9: Qualitative comparison of contours of streamwise velocity disturbance $\mathrm{u}$ in the $(x, z)$-plane (light shades of gray correspond to high values).

organized as follows: Section 1 is a background review; Section 2 shows the case set up and code validation; Section 3 is our observation and analysis; Section 4 is a summary of our new finding; Section 5 is the conclusions which are made based on our recent DNS.

1.8. New Theory on Boundary Layer Transition Given by Liu. Classical theory on boundary layer transition can be described four stages: (1) boundary layer receptivity; (2) linear instability; (3) non-linear growth; (4) vortex breakdown to turbulence. Apparently, we disagree with the classical 


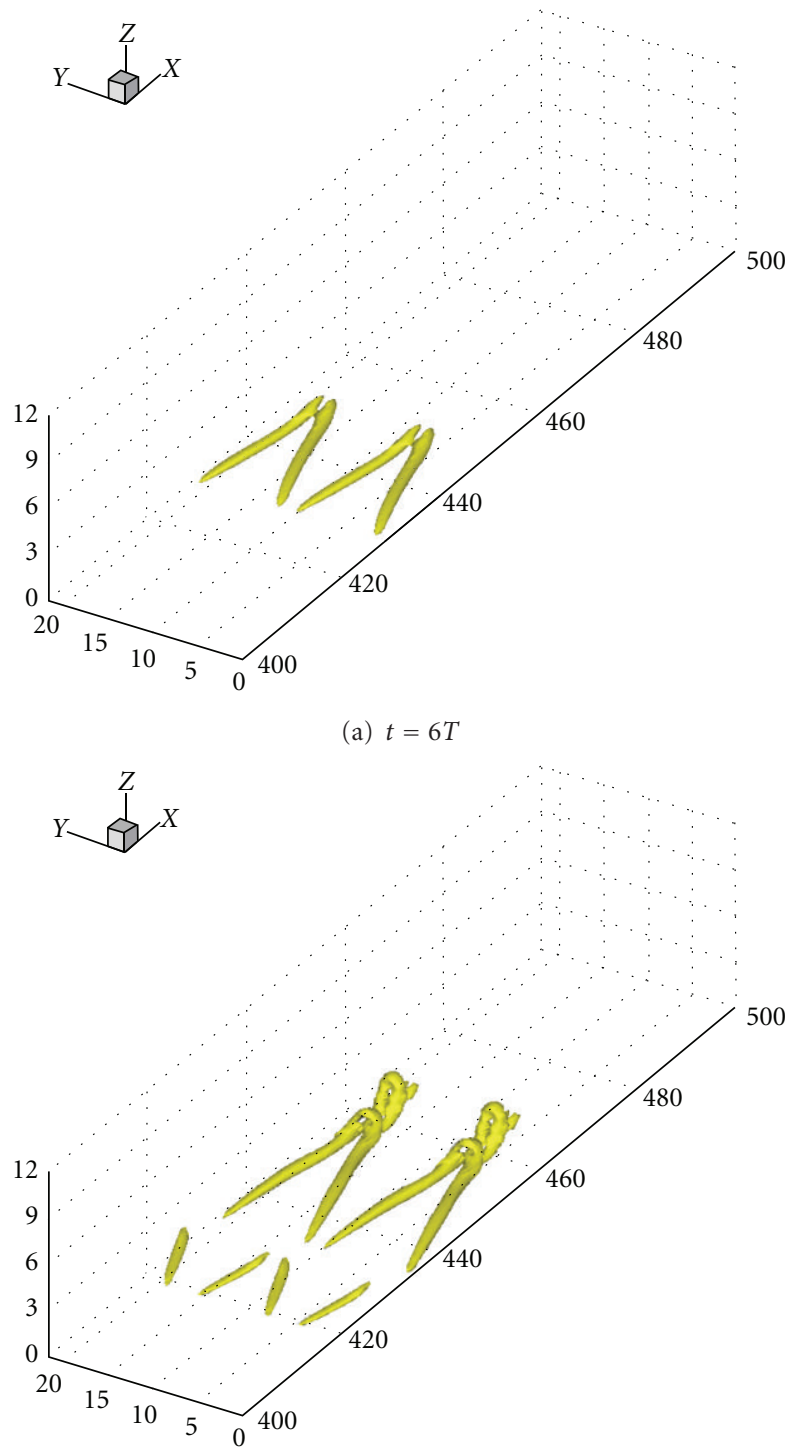

(c) $t=6.4 T$



(b) $t=6.2 \mathrm{~T}$

$$
r V^{X}
$$

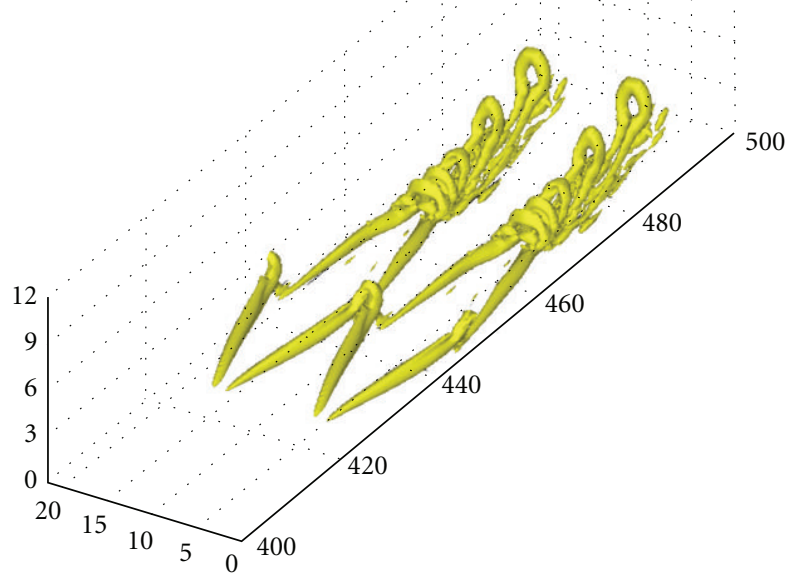

(d) $t=7.0 \mathrm{~T}$

Figure 10: Evolution of vortex structure at the late-stage of transition (where $T$ is the period of $T$-S wave).

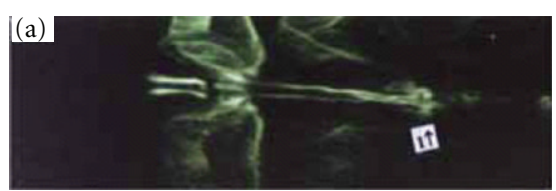

(a)

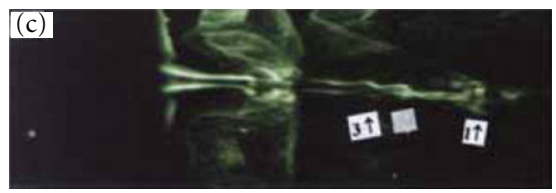

(c)

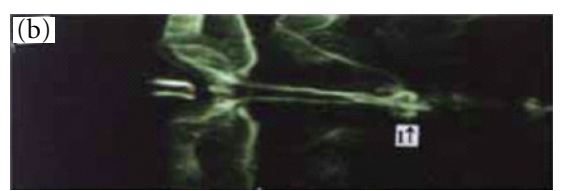

(b)

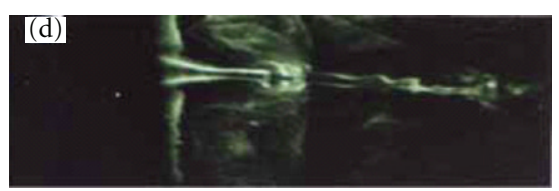

(d)

FIGURE 11: Evolution of the ring-like vortex chain by experiment [5]. 


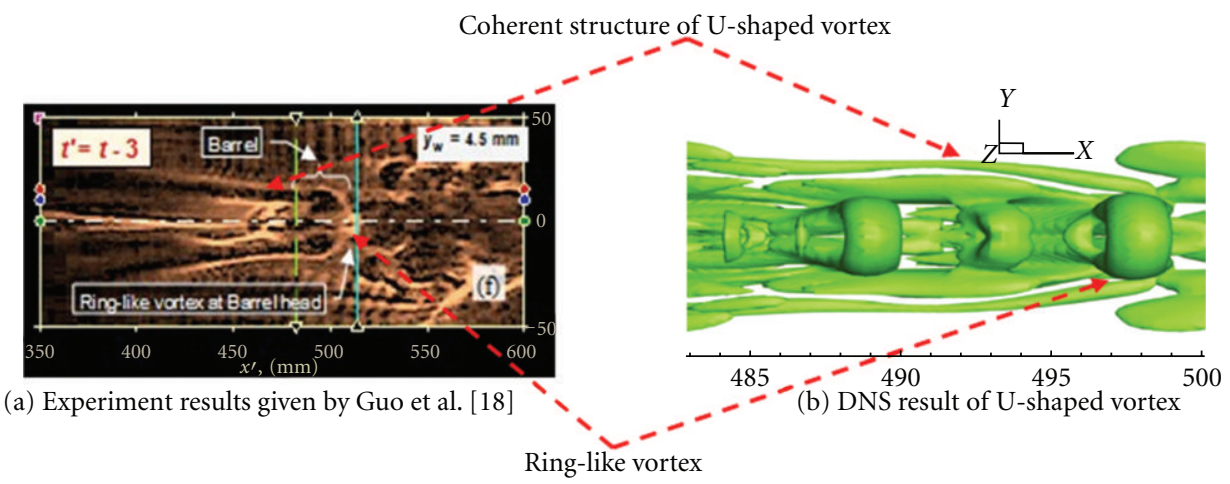

FIGURE 12: Qualitative vortex structure comparison with experiment.

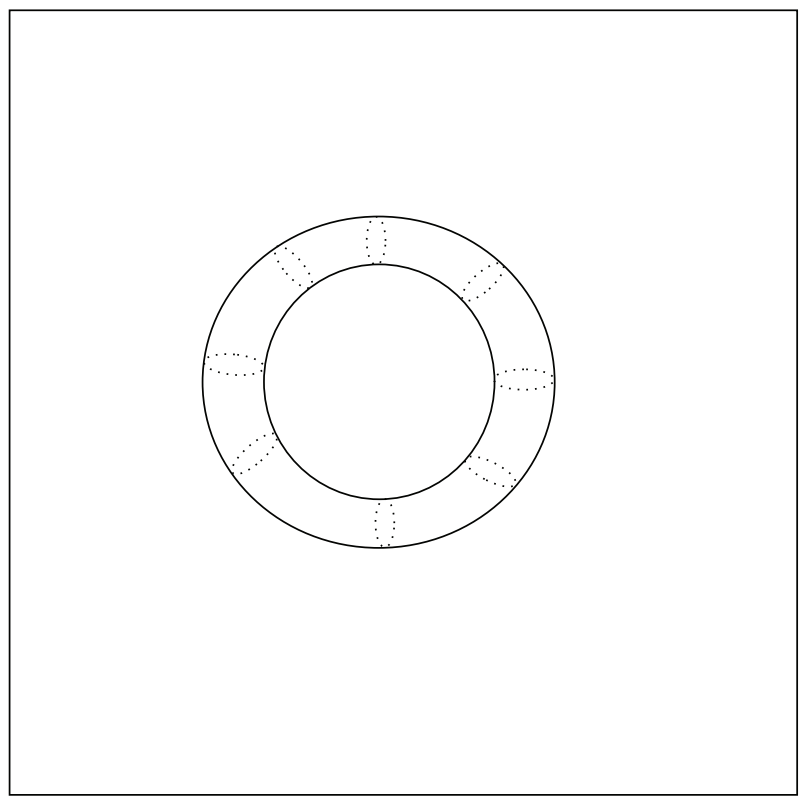

(a) Vortex ring without legs

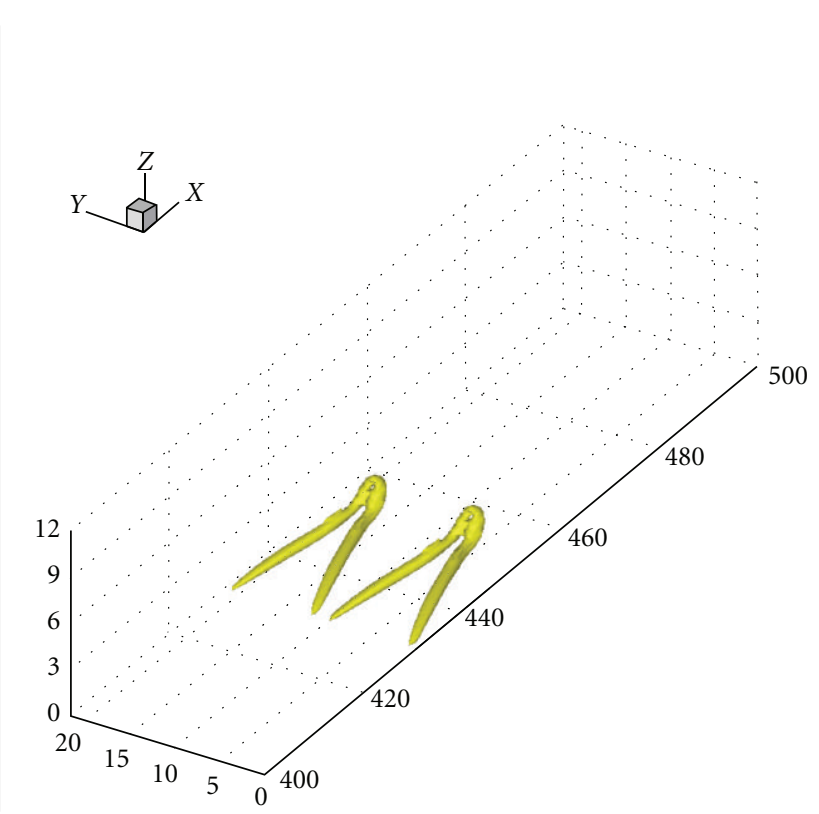

(b) Vortex ring with legs

FiguRE 13: Vortex rings with or without legs.

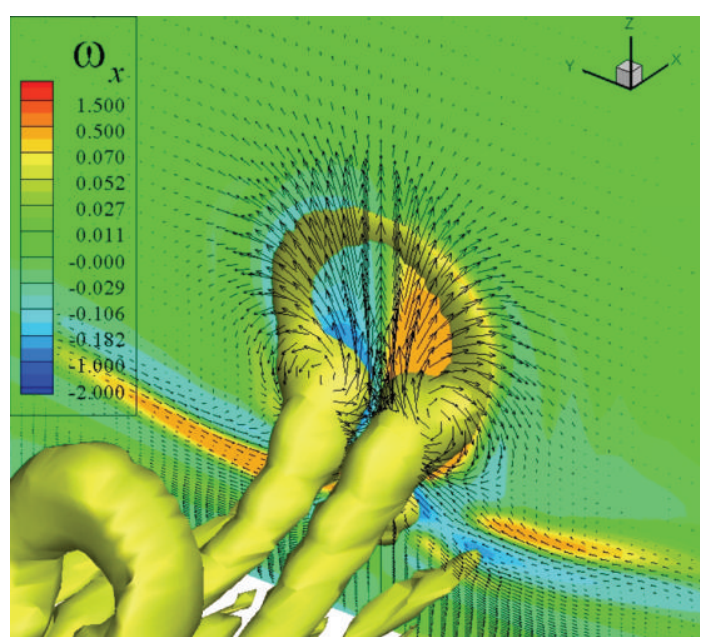

(a)

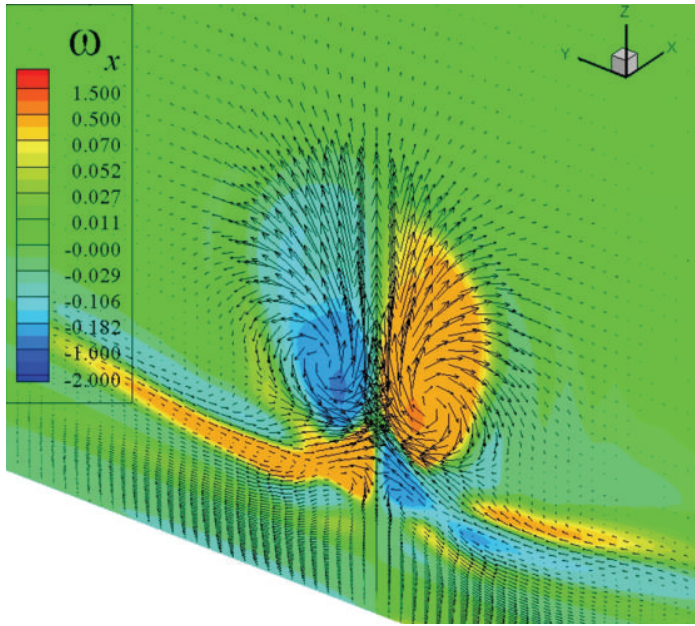

(b)

FIGURE 14: Formation processes of first ring-like vortex. 


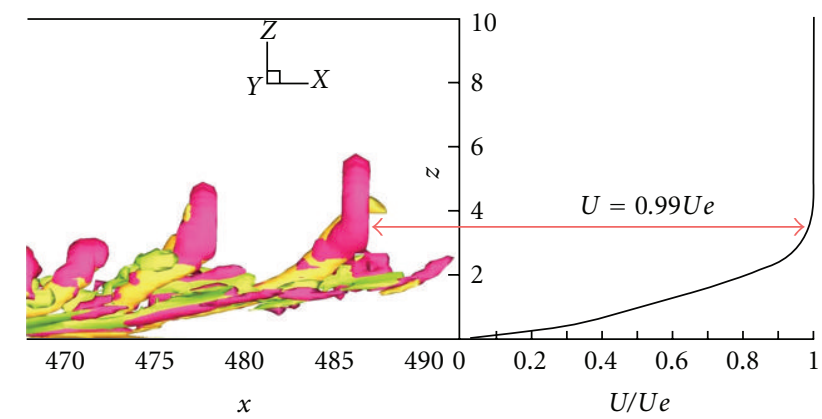

FIGURE 15: The shape and position of ring-like vortices in boundary layer $(z=3.56, U=0.99 U e)$.

theory on "vortex breakdown to turbulence." The new theory of boundary layer transition can be described by five stages: (1) boundary layer receptivity; (2) linear instability; (3) large vortex structure formation; (4) small vortices generation; (5) symmetry loss and turbulence formation. By the way, the vortex cascade in turbulence given by Richardson, Kolmogorov and others is not observed.

1.9. New Theory on Turbulence Generation and Sustenance Given by Liu. The new theory on turbulence formation and sustenance can be described as follows. All small length scales (turbulence) are generated by shear layer instability which is produced by large vortex structure with multiple level vortex rings, multiple level sweeps and ejections, and multiple level negative and positive spikes near the laminar sublayers. Therefore, "turbulence" is not generated by "vortex breakdown" but rather positive and negative spikes and consequent high shear layers. "Shear layer instability" is considered as the "mother of turbulence." This new theory may give a universal mechanism for turbulence generation and sustenance-the energy is brought by large vortex structure through multiple level sweeps in a boundary layer.

\section{Case Setup and Code Validation}

2.1. Case Setup. The computational domain is displayed in Figure 1. The grid level is $1920 \times 128 \times 241$, representing the number of grids in streamwise $(x)$, spanwise $(y)$, and wall normal $(z)$ directions. The grid is stretched in the normal direction and uniform in the streamwise and spanwise directions. The length of the first grid interval in the normal direction at the entrance is found to be 0.43 in wall units $\left(Y^{+}=0.43\right)$. The parallel computation is accomplished through the message passing interface (MPI) together with domain decomposition in the streamwise direction (Figure 2). The flow parameters, including Mach number and Reynolds number are listed in Table 1. Here, $x_{\text {in }}$ represents the distance between leading edge and inlet, $L x, L y, L z_{\text {in }}$ are the lengths of the computational domain in $x-, y$-, and $z$-directions, respectively, and $T_{w}$ is the wall temperature.

2.2. Code Validation. The DNS code- "DNSUTA" has been validated by NASA Langley and UTA researchers $[24,32,34]$ carefully to make sure the DNS results are correct.
TABLe 1: Flow parameters.

\begin{tabular}{cccccccc}
\hline$M_{\infty}$ & $\operatorname{Re}$ & $x_{\text {in }}$ & $L x$ & $L y$ & $L z_{\text {in }}$ & $T_{w}$ & $T_{\infty}$ \\
\hline 0.5 & 1000 & $300.79 \delta_{\text {in }}$ & $798.03 \delta_{\text {in }}$ & $22 \delta_{\text {in }}$ & $40 \delta_{\text {in }}$ & $273.15 \mathrm{~K}$ & $273.15 \mathrm{~K}$ \\
\hline
\end{tabular}

2.2.1. Comparison with Linear Theory. Figure 3 compares the velocity profile of the $T-S$ wave given by our DNS results to linear theory. Figure 4 is a comparison of the perturbation amplification rate between DNS and LST. The agreement between linear theory and our numerical results is quite good.

2.2.2. Grid Convergence. The skin friction coefficient calculated from the time-averaged and spanwise-averaged profile on a coarse and fine grid is displayed in Figure 5. The spatial evolution of skin friction coefficients of laminar flow is also plotted out for comparison. It is observed from these figures that the sharp growth of the skin-friction coefficient occurs after $x \approx 450 \delta_{\text {in }}$, which is defined as the "onset point." The skin friction coefficient after transition is in good agreement with the flat-plate theory of turbulent boundary layer by Cousteix in 1989 [35]. Figures 5(a) and 5(b) also show that we get grid convergence in skin friction coefficients.

2.2.3. Comparison with Log Law. Time-averaged and spanwise-averaged streamwise velocity profiles for various streamwise locations in two different grid levels are shown in Figure 6. The inflow velocity profiles at $x=300.79 \delta_{\text {in }}$ is a typical laminar flow velocity profile. At $x=632.33 \delta_{\text {in }}$, the mean velocity profile approaches a turbulent flow velocity profile (log law). This comparison shows that the velocity profile from the DNS results is a turbulent flow velocity profile and the grid convergence has been realized.

2.2.4. Spectra and Reynolds Stress (Velocity) Statistics. Figure 7 shows the spectra in $x$ - and $y$-directions. The spectra are normalized by $z$ at location of $\operatorname{Re}_{x}=1.07 \times$ $10^{6}$ and $y^{+}=100.250$. In general, the turbulent region is approximately defined by $y^{+}>100$ and $y / \delta<0.15$. In our case, The location of $y / \delta=0.15$ for $\operatorname{Re}_{x}=1.07 \times 10^{6}$ is corresponding to $y^{+} \approx 350$, so the points at $y^{+}=100$ and 250 should be in the turbulent region. A straight line with slope of $-3 / 5$ is also shown for comparison. The spectra tend to tangent to the $k^{-5 / 3}$ law.

Figure 8 shows Reynolds shear stress profiles at various streamwise locations, normalized by square of wall shear velocity. There are 10 streamwise locations which are selected starting from leading edge to trailing edge. As expected, close to the inlet at $\mathrm{Re}_{x}=326.8 \times 10^{3}$ where the flow is laminar, the values of the Reynolds stress is much smaller than those in the turbulent region. The peak value increases with the increase of $x$. At around $\mathrm{Re}_{x}=432.9 \times 10^{3}$, a big jump is observed, which indicates the flow is in transition. After $\operatorname{Re}_{x}=485.9 \times 10^{3}$, the Reynolds stress profile becomes close to each other in the turbulent region. So for this case, we can consider that the flow transition starts after $\operatorname{Re}_{x}=$ $490 \times 10^{3}$. 


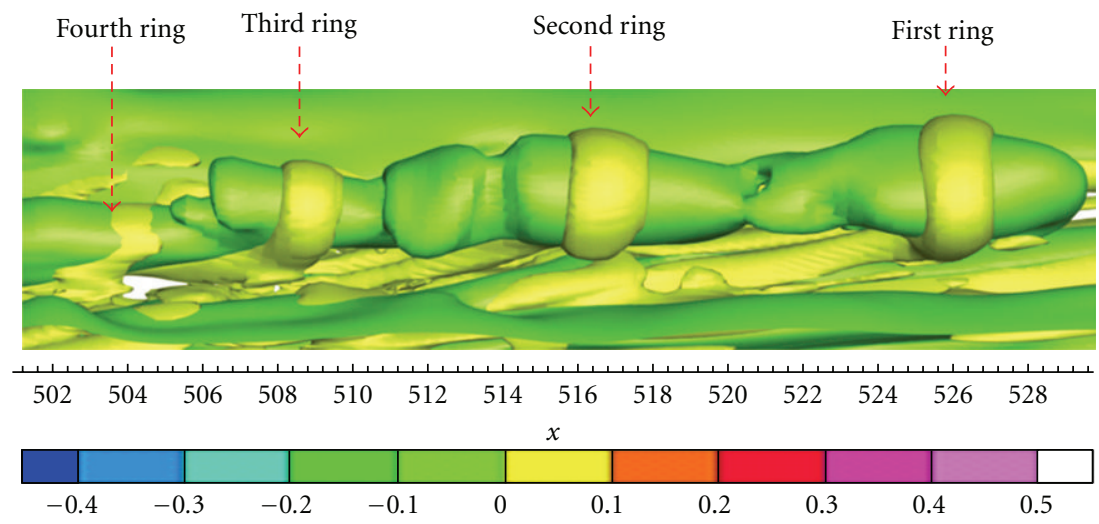

FIgURE 16: Three-dimensional momentum deficit and multiple ring-like vortex formation.
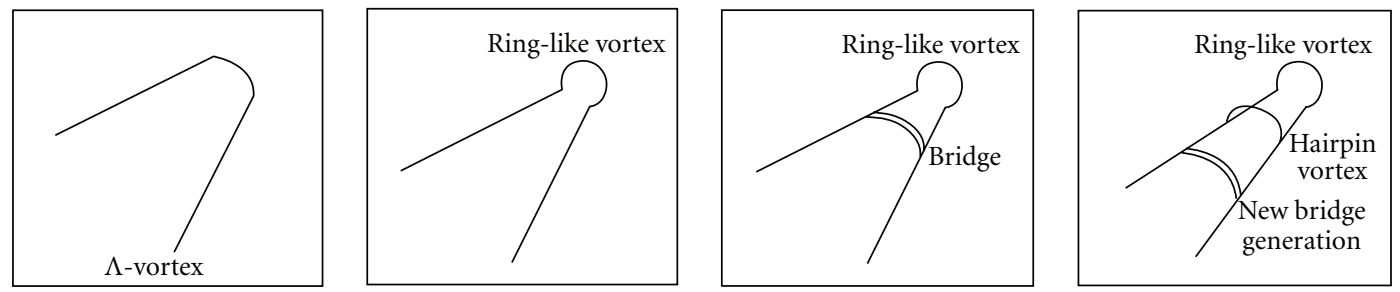

(a)
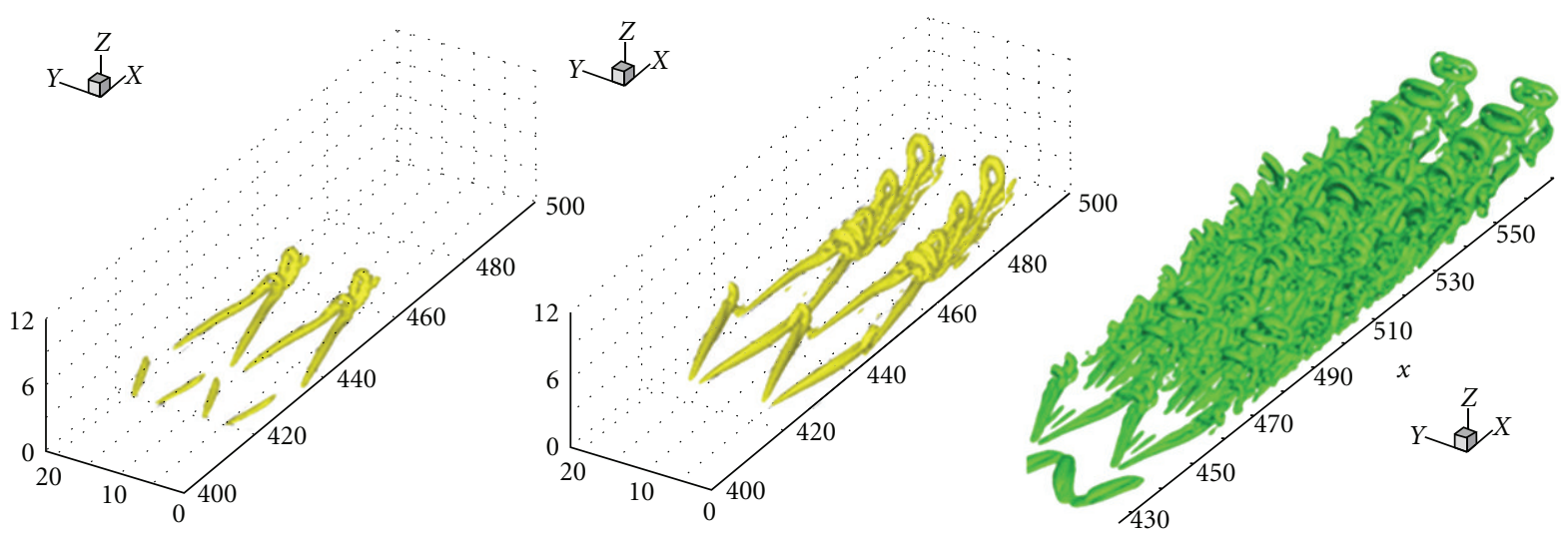

(b)

FIGURE 17: (a) Sketch for mechanism of multiring generation, (b) multiple ring formation.

2.2.5. Comparison with Other DNS. Although we cannot compare our DNS results with those given by Borodulin et al. [4] quantitatively, we still can find that the shear layer structure are very similar in two DNS computations in Figure 9.

2.2.6. Comparison with Experiment. By this $\lambda_{2}$-eigenvalue visualization method, the vortex structures shaped by the nonlinear evolution of $T$ - $S$ waves in the transition process are shown in Figure 10. The evolution details are briefly studied in our previous paper [20] and the formation of ring-like vortices chains is consistent with the experimental work ([5], Figure 11) and previous numerical simulation by Bake and his coauthors [15].
2.2.7. U-Shaped Vortex in Comparison with Experimental Results. Figure 12(a) [19] represents an experimental investigation of the vortex structure including ring-like vortex and barrel-shaped head (U-shaped vortex). The vortex structures of the nonlinear evolution of $T-S$ waves in the transition process are given by DNS in Figure 12(b). By careful comparison between the experimental work and DNS, we note that the experiment and DNS agree with each other in a detailed flow structure comparison. This cannot be obtained by accident, but provides the following clues: (1) both DNS and experiment are correct, (2) disregarding the differences in inflow boundary conditions (random noises versus enforced $T-S$ waves) and spanwise boundary conditions (nonperiodic versus periodic) between experiment and DNS, the vortex 


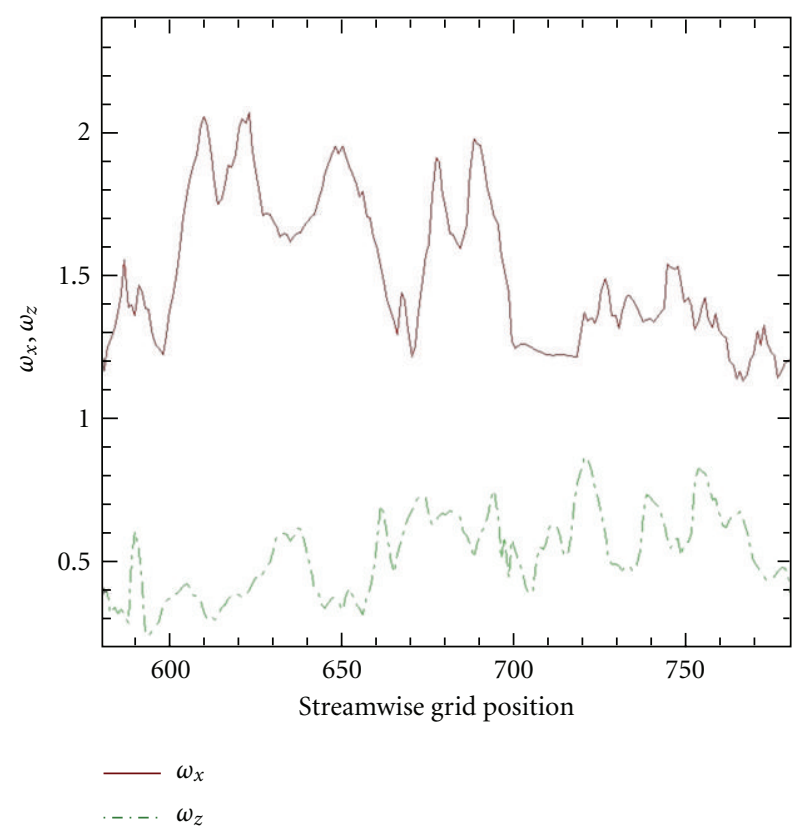

Figure 18: Maximum streamwise and spanwise vorticity along $x$ coordinate (Helmoholtz vorticity conservation).

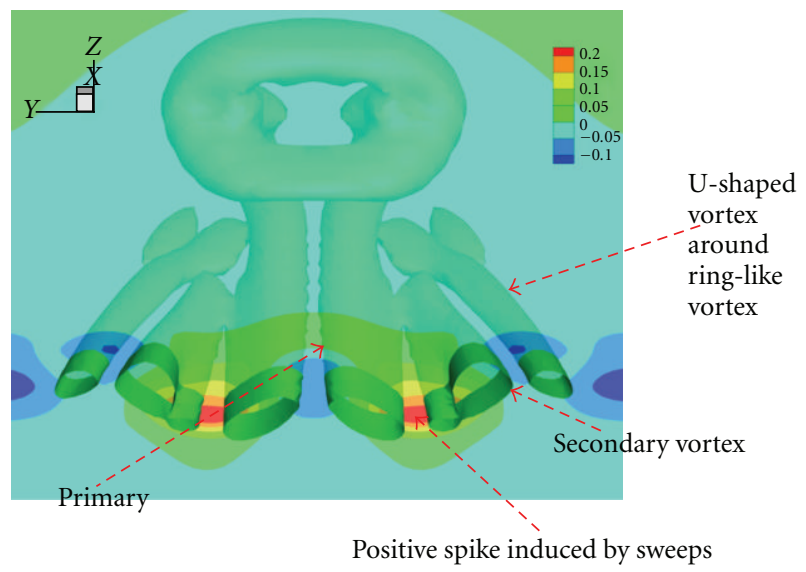

FIGURE 19: U-shaped vortex with streamwise velocity perturbation contour.

structures are same, (3) no matter K-, $\mathrm{H}$ - or other types of transition, the final vortex structures are same, (4) there is a universal structure for late boundary layer transition; (5) turbulence has certain coherent structures (CS) for generation and sustenance.

All these verifications and validations above show that our code is correct and our DNS results are reliable.

\section{Observation and Analysis done by Our DNS}

Following observations have been made and reported by our previous publications $[20-28,30-32,36,37]$.

3.1. Mechanism of Large Coherent Vortex Structure. Late boundary transition starts from the formation of the first vortex ring.

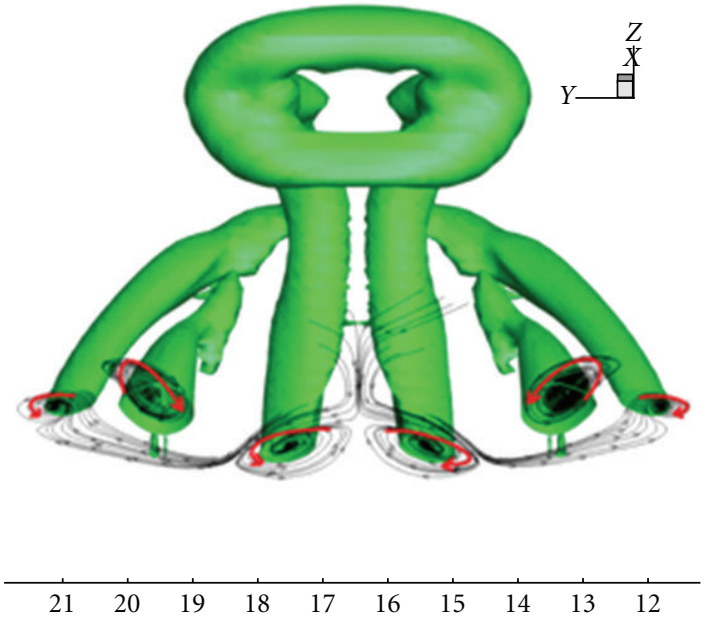

FIgURE 20: Isosurface of $\lambda_{2}$ and stream trace at $x=530.348 \delta_{\text {in }}$.

3.1.1. Vortex Rings in Flow Field [23]. According to Helmholtz vorticity conservation law, the vortex tube cannot have ends inside the flow field. The only form of vortex tube existing inside the flow filed must be ring with or without legs (Figure 13).

3.1.2. Mechanism of First Ring-Like Vortex Formation [23]. The ring shape of first ring-like vortex is caused by the interaction between the prime streamwise vortices and secondary streamwise vortices (Figure 14), which is quite different from the one given by Moin et al. [38]. The ring-like vortex is located at the edge of the boundary layer or in other words located in the inviscid zone $(z=3.56, U=0.99 U e)$. The vortex ring is perpendicularly standing and the shape of the ring is almost perfectly circular, which will generate the strongest downwash sweeps (Figures 14 and 15). More important, no vortex "pinch off" is observed.

3.1.3. Mechanism of Multiple Ring Formation [37]. The ejection by rotation of the primary vortex legs brings low speed flow from lower boundary layer up and forms a cylinder-like momentum deficit zone in the middle of the two legs (green in Figure 16). The momentum deficit zone is also located above the legs and forms shear layers due to the stream-wise velocity difference between surrounding highspeed flow and low-speed flow in the deficit zone. These shear layers are not stable and multiple ring-like vortices (yellow in Figure 16) are generated by the shear layers one by one (Figure 17). This process must satisfy the Helmholtz vorticity conservation and the primary streamwise vorticity must be reduced when a new spanwise-oriented ring is formed (Figure 18). Different from Borodulin et al. [4], neither "vortex breakdown and reconnection" nor "Crow theory" is observed by our new DNS.

3.1.4. Primary, Secondary, and U-Shaped Vortices [31]. Ushaped vortex is part of large coherent vortex structure and is generated by secondary vortices which are induced by the 


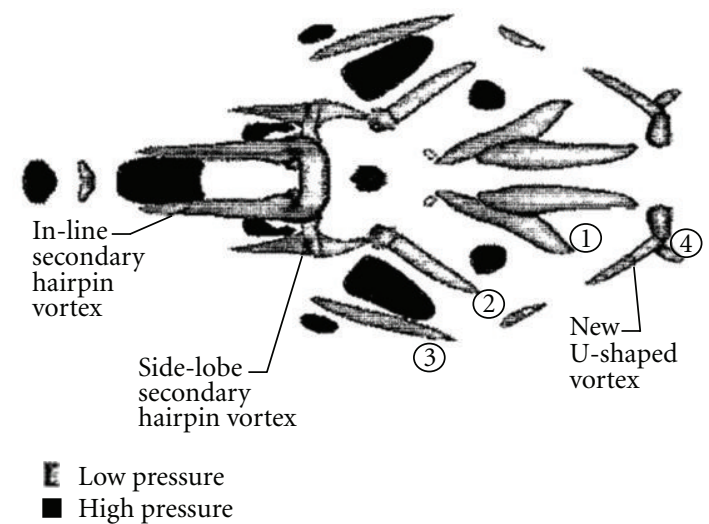

(a)

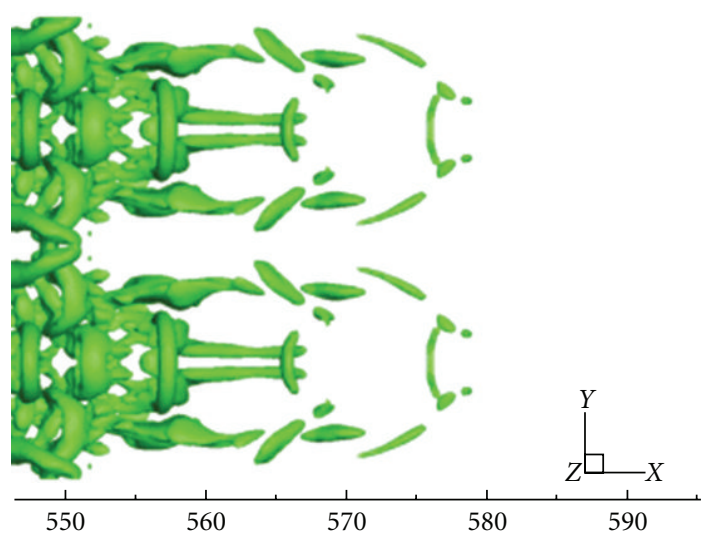

(b)

Figure 21: (a) U-shaped vortex [9], (b) DNS with a different $\lambda$-value (fake).

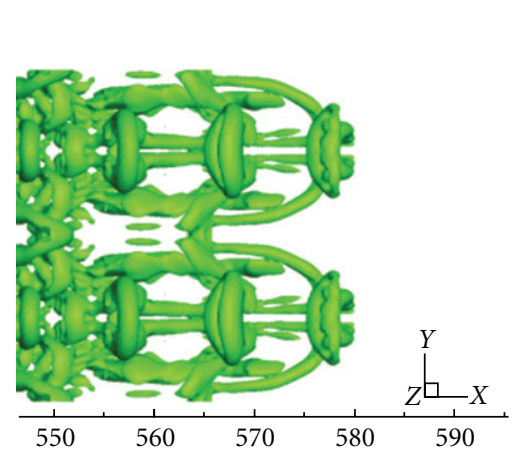

(a) Top view

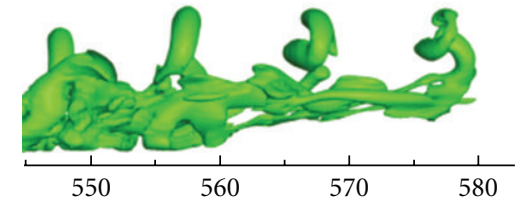

(b) Side view

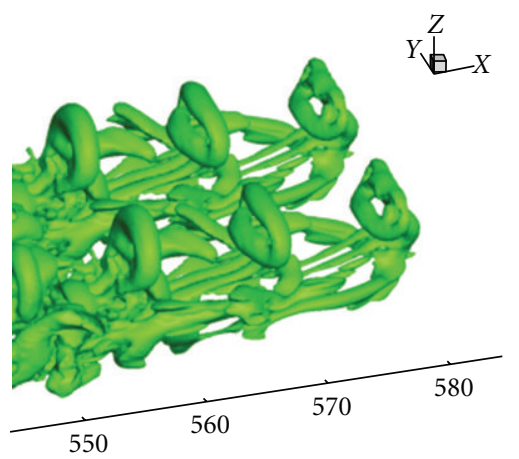

(c) Angle view

Figure 22: View of young turbulence spot head from different directions $(t=8.8 T)$.

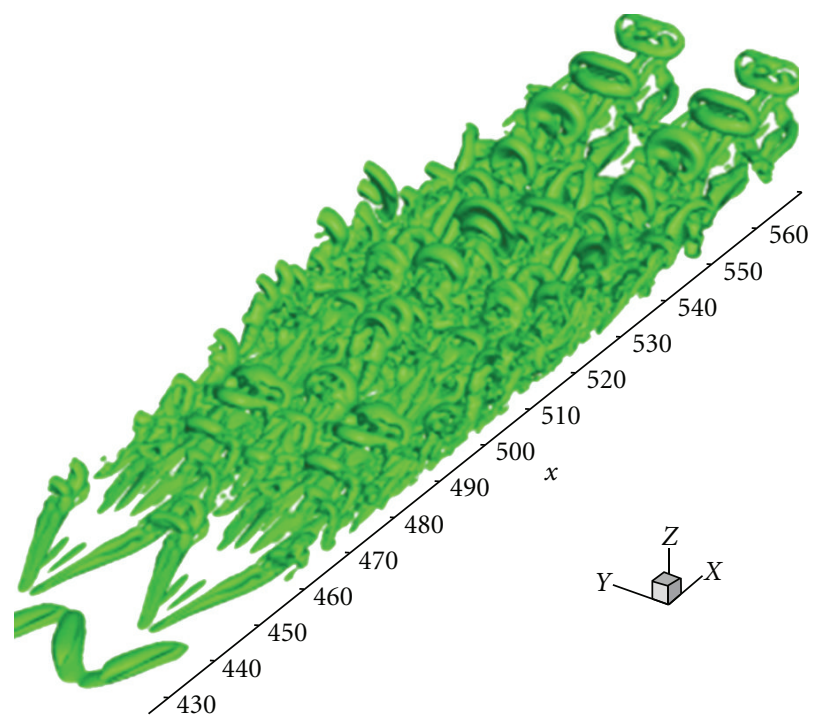

(a) Angle view

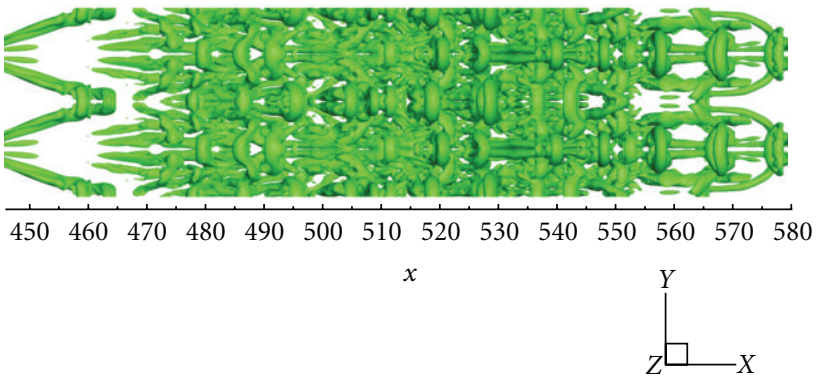

(b) Top view

FIgURE 23: 3D angle view and 2D top view of the young turbulence spot $(t=8.8 T)$. 


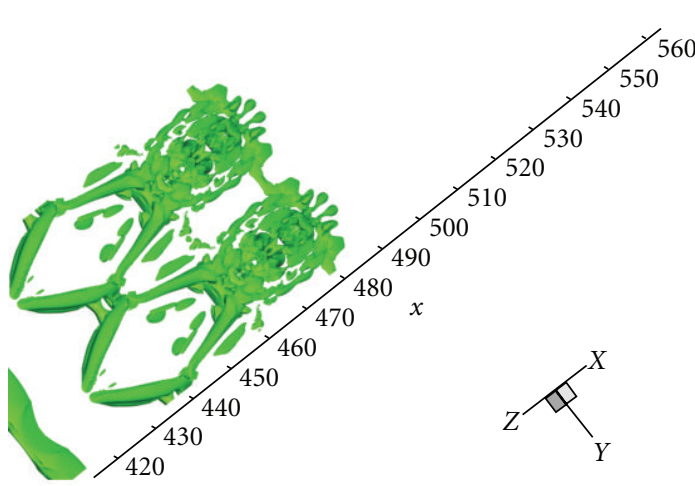

(a) $t=7.0 T$

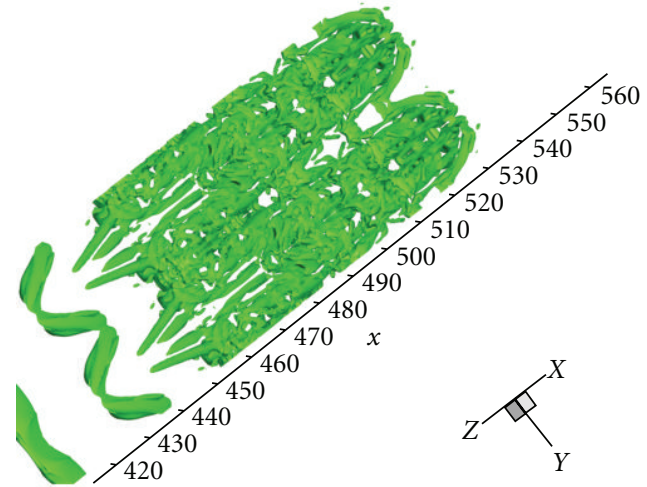

(b) $t=8.0 \mathrm{~T}$

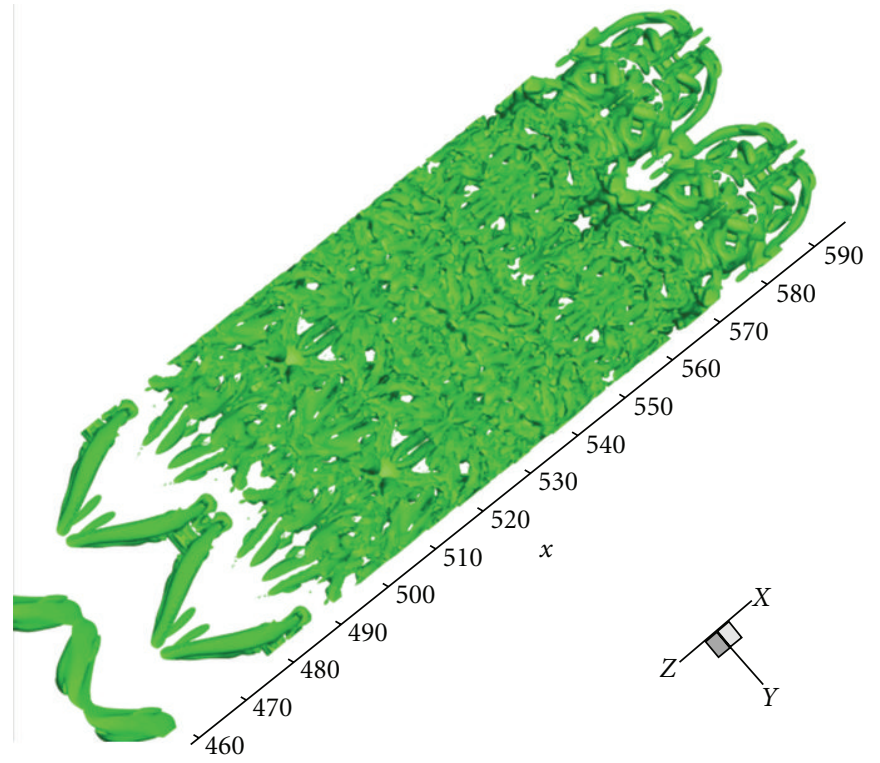

(c) $t=8.8 \mathrm{~T}$

Figure 24: Small length scale vortex generation at different time steps (view up from bottom): small length scale vortices are generated by the solid wall near the ring necks from the beginning to the end.

primary vortex. Being different from Guo et el. [19], the Ushaped vortex is not a wave and is not induced by second sweeps and positive spikes. Actually, the U-shaped vortex is a tertiary (not secondary) vortex with same vorticity sign as the original ring legs (Figures 19 and 20). In addition, the U-shaped vortices serve as additional channels to supply vorticity to the multiple ring structure [32].

3.2. Mechanism of Small Length Vortices Generation. Turbulence has two futures: (1) small length vortices; (2) nonsymmetric structure (Randomization).

3.2.1. "Vortex Breakdown to Turbulence" is Challenged [29, $30]$. In the new DNS $[27,28]$, it has been observed that the multiple vortex structure is a quite stable structure and never breaks down. Previously reported "vortex breakdown" is either based on 2-D visualization or made by using low pressure center as the vortex center (Figure 21(a)). We can use a different $\lambda_{2}$ value to get similar "vortex breakdown"
(Figure 21(b)), which is faked. Here, we define vortex as a tube with a rotated core and isosurfaces of constant vorticity flux. However, there is no evidence that the vortex breaks down. Let us look at the head of the so-called "turbulence spot" from different directions of view (Figure 22). Due to the increase of the ring (bridge) number and vorticity conservation, the leading rings will become weaker and weaker until they cannot be detected, but they never break down (see Figure 23).

\subsubsection{Mechanism of Small-Length Scale (Turbulence)} Generation-by "Shear Layers" [30, 32]. The question will be raised that where do the small length scales come from if the small vortices are not generated by "vortex breakdown"? Since we believe that the small vortices are generated by shear layers near the wall surface, we take snap shots in the direction of view from the bottom to top (Figure 24). The evidence provided by our new DNS confirms that the small length scale vortices are generated near the bottom of the 


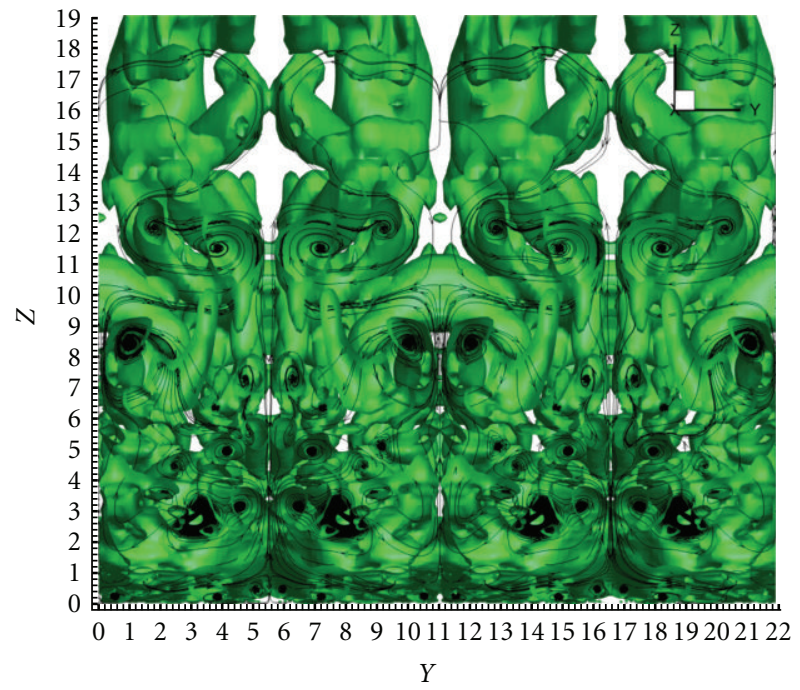

(a) $\lambda_{2}$ isosurfaces

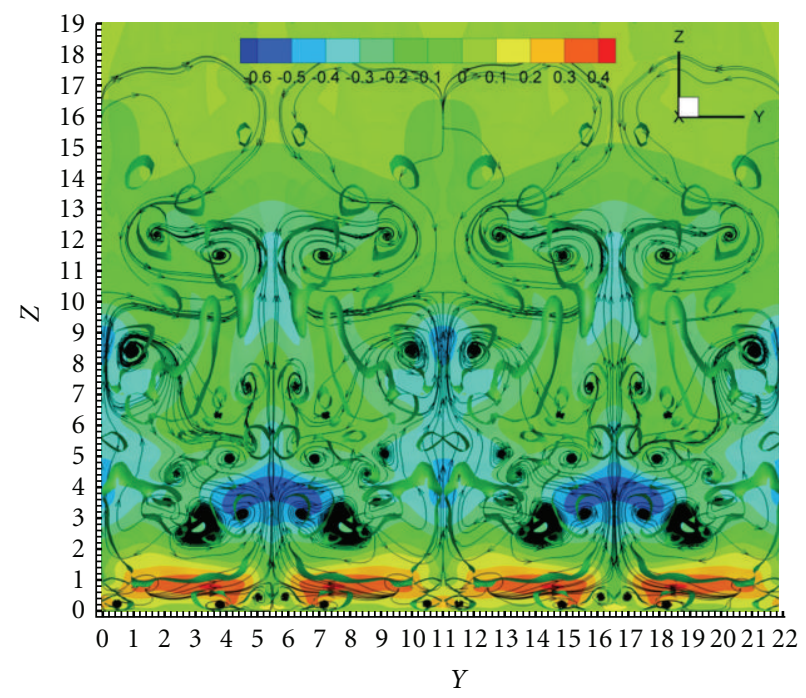

(b) Contour of velocity perturbation

FIGURE 25: Visualization of isosurface $\lambda_{2}$ and velocity perturbation at $x=508.633$ for $(Y, Z)$-Plane $t=15 T$.

boundary layer. Being different from classical theory which believes turbulence is generated by "vortex breakdown", our new DNS found that all small length scales (turbulence) are generated by high-shear layers (HS) near the bottom of the boundary layers. There is no exception. When we look at the later stage of flow transition at $t=15 T$, we can see that all small length scales are located around the high-shear layers, especially at those near the wall surface (Figures 25 and 26).

3.2.3. High-Shear Layer Formation by Sweeps [30]. Experiment and our numerical results have confirmed that there is a second sweep excited by every ring-like vortex (Figure 27). Combined with the first sweep generated by the original $\lambda$ vortex legs, it forms strong positive spikes which generate strong high shears at the bottom of the boundary layer (red in Figure 28). Figures 29 and 30 are contours of isosurface of $\lambda_{2}$ and velocity perturbation at $x=508.66$. The second sweep movement induced by ring-like vortices working together with first sweep will lead to huge energy and momentum transformation from high-energy inviscid zones to lowenergy zones near the bottom of the boundary layers and we can observe that all small length scales are generated around high shear layers between the positive spike (momentum increment) and solid wall surface.

\subsubsection{Multiple-Level Sweeps and Multiple-Level Negative} and Positive Spikes [32]. The positive spikes (momentum increment) could generate new ring-like vortices. The new ring-like vortices can further generate new sweeps and form new positive spikes (Figure 31) at the location which are very close to the bottom of the boundary layer (laminar sublayer). The new positive spike could induce new smaller vortex rings by unstable shear layers. These multilevel sweeps and multilevel negative and positive spikes provide channels for energy transfer from the inviscid area (high energy area) down to the bottom (low energy area). This is the mechanism why turbulence can be generated and sustained. This mechanism can be interpreted as a universal mechanism for both transitional and turbulent flow in a boundary layer.

\subsubsection{Energy Transfer Paths and Universal Turbulence Spot} Structure [32]. Figures 32 and 33 are sketches describing energy transfer and likely universal turbulence structure.

3.2.6. Surface Friction. Figure 34 is a time and spanwise averaged surface friction coefficient (CF). From the figure, it is clearly seen that there is a jump starting at $x=430$ which indicates an onset of flow transition and reach a maximum value at $x=508.663$. It is conventionally believed that the CF is large in the turbulent area and small in the laminar area due to the strong mixing in turbulent flow. That is the reason why most turbulence models are formulated based on change of turbulent viscosity. However, from Figure 34, it is easy to find that the CF reaches maximum in a laminar area and is not directly related to mixing. Further analysis found the viscosity is not changed for incompressible flow, but the shear layer is changed sharply when the first and second sweeps caused positive spikes (momentum increment), HS layers and consequent small vortices generations. The velocity gradients suddenly jump to a very large level in the laminar sub-layer and then the CF becomes very large. Therefore, the CF jump is not caused by mixing or viscosity coefficients increase, but is, pretty much, caused by velocity gradient jump due to small vortices formation.

3.3. Loss of Symmetry (Randomization). The existing theory [41] believes that the flow randomization in DNS is caused by the background noises and removal of periodic boundary conditions. They believe that randomization starts from loss of symmetry, which is caused by background noise. According to their theory, the ring tip is affected first, which rapidly influences the small length scale structure. This will 


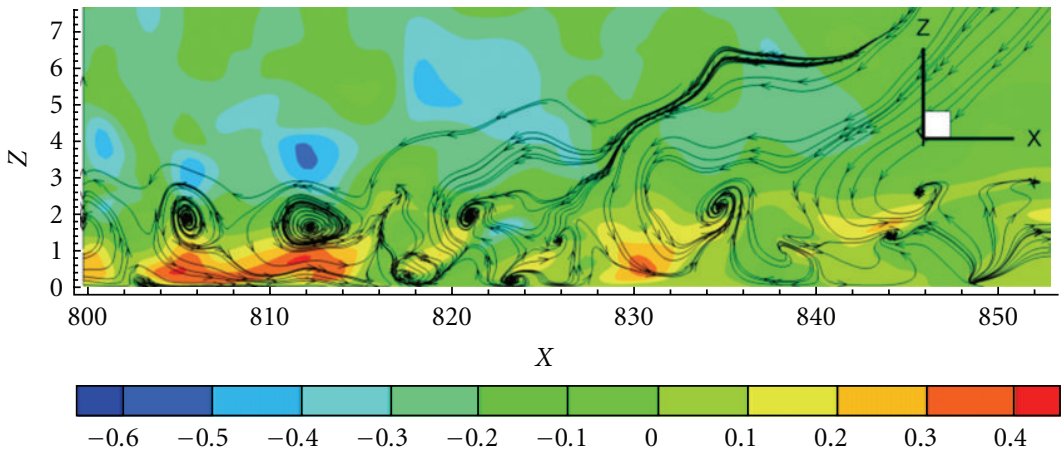

Figure 26: Visualization of isosurface $\lambda_{2}$ and velocity perturbation at $y=4$ for $(X, Z)$-Plane $t=15 T$.

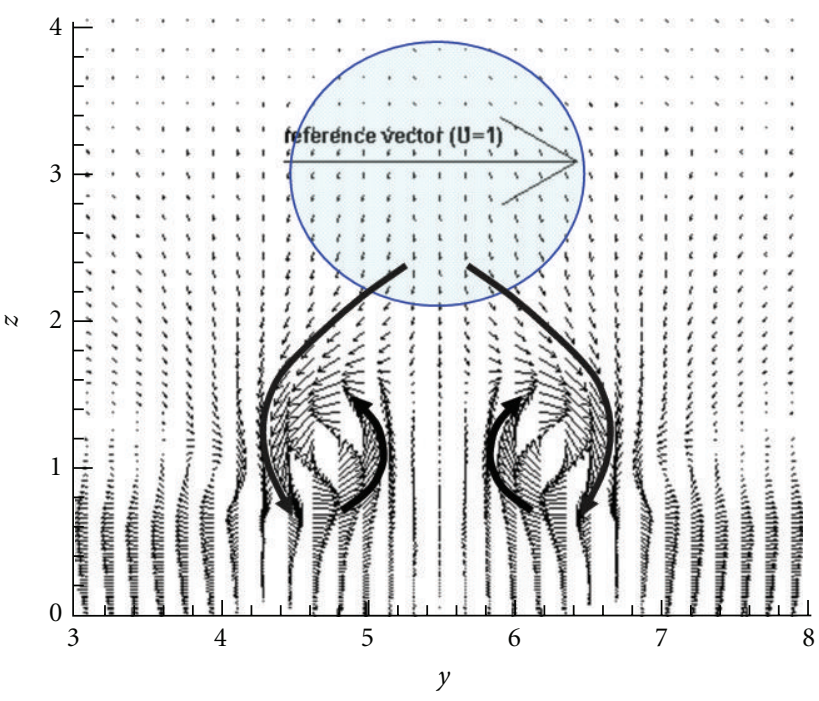

FIGURE 27: Velocity vector field.
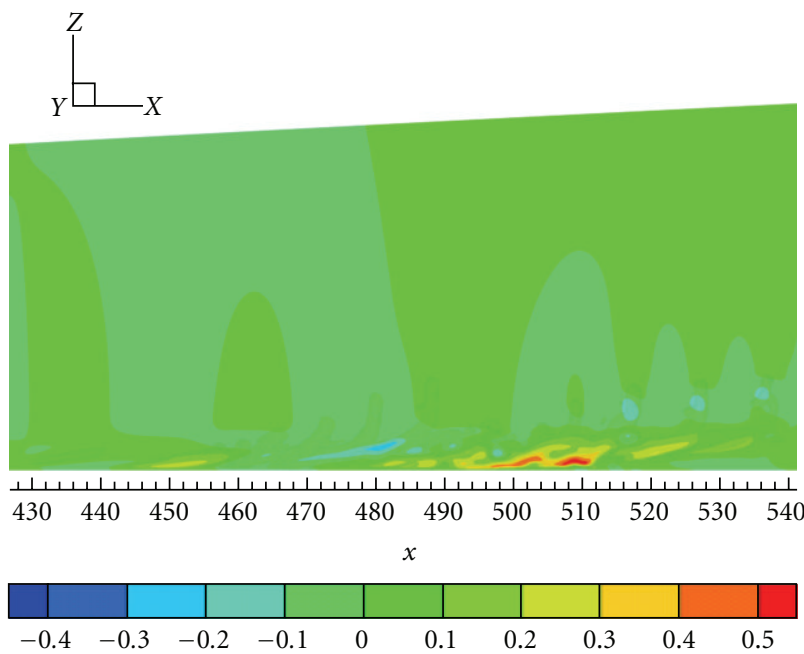

FIGURE 28: Vorticity distribution at $x=508.633$.

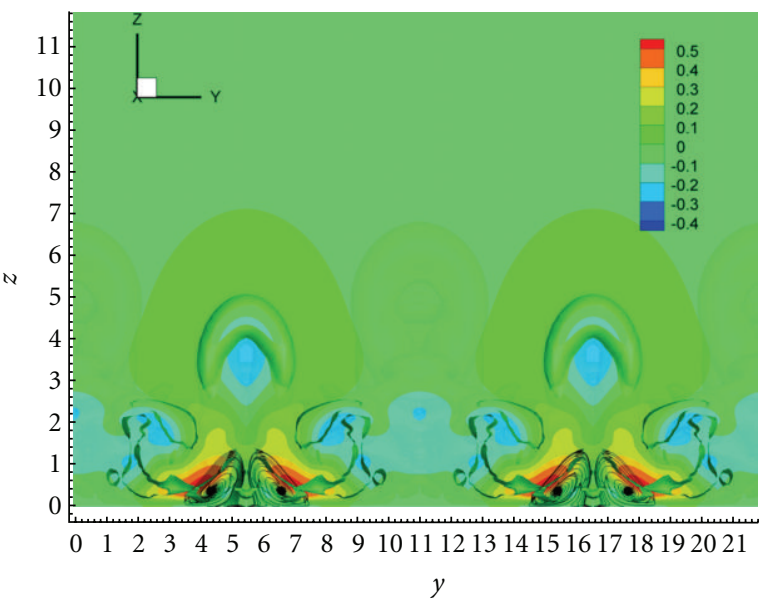

FIGURE 29: :Isosurface of $\lambda_{2}$ and velocity at $x=508.66$.

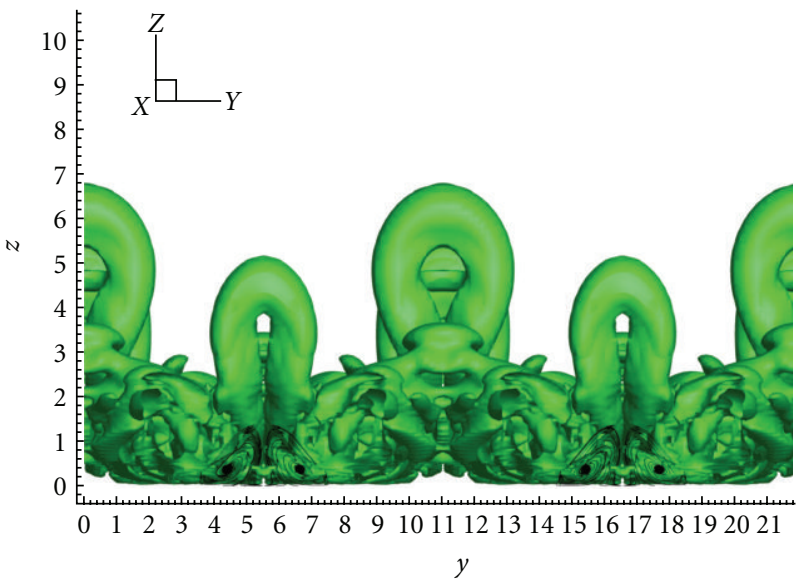

FIgURE 30: Isosurface of $\lambda_{2}$ and stream traces at $x=508.66$.

lead to loss of symmetry and randomization for the whole flow field quickly.

However, what we observed in our new DNS is that the loss of symmetry starts from second level rings (Figure 35) while the top and bottom rings are still symmetric 


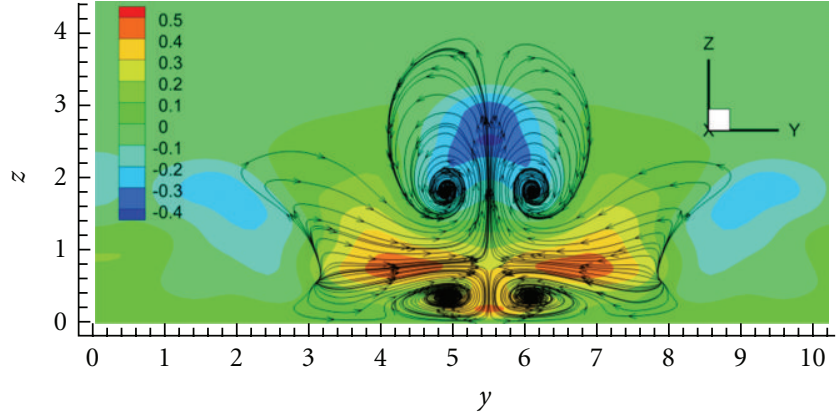

(a) Streamwise velocity perturbation and stream trace

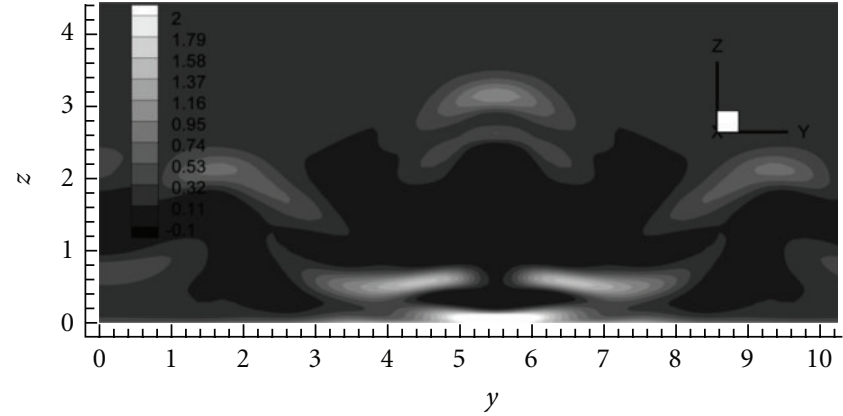

(b) Spanwise vorticity distribution

FIGURE 31: (a) Multilevel sweeps; (b) multilevel positive spikes.

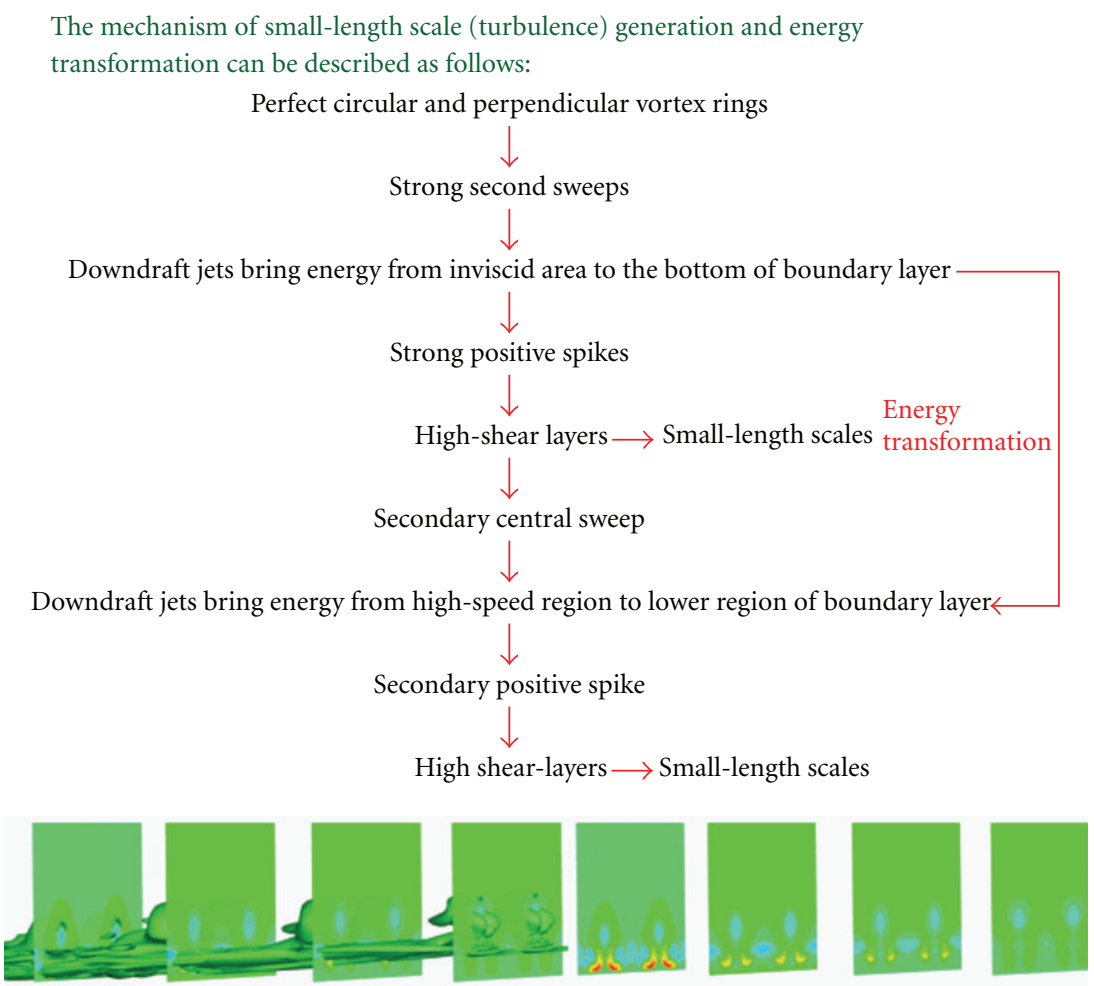

There is no positive spike for the leading primary rings which are skewed and sloped.

FIgURE 32: Energy transfer paths and universal structure for turbulence.

(Figure 36). The nonsymmetric structure of second level rings will influence the small length scale at the bottom quickly. The change of symmetry in the bottom of the boundary layer is quickly spread to the up level through ejections. This will lead to randomization of the whole flow field. Therefore, the internal instability of multiple level ring spot overlapping structure, especially the middle ring cycles, is a critical reason for flow randomization, but mainly not the background noise. In addition, the loss of symmetry starts in the middle of the flow domain, not the inflow and not the outflow (Figure 37(a)). As mentioned, we did not change the periodic boundary conditions and the solution is still periodic in the spanwise direction (period $=2 \pi$ is enforced). In addition, we did not add any additional background noise or inflow perturbation. However, we found that the flow lost symmetry first and then was randomized step by step (see Figures 38(a)-38(d)). The flow was first periodic (period $=\pi$ ) and symmetric in the spanwise direction, $\sum_{k=0}^{n} a_{k} \cos (2 k y)$, but then lost symmetry in some areas, and finally everywhere. It was periodic with a period of $\pi$, same as the inflow, but changed with a period of $2 \pi$. The flow is still periodic because we enforced the periodic boundary condition in the spanwise direction with a period of $2 \pi$. This means the flow does not only have $\sum_{k=0}^{n} a_{k} \cos (2 k y)$ but also has $\sum_{k=0}^{n} b_{k} \sin (2 k y)$ which is newly generated. Meanwhile, flow lost periodicity with period $=\pi$, but has to be periodic with period $=2 \pi$ (Figures $38(\mathrm{c})$ and $38(\mathrm{~d})$ ), which we enforced. Since the DNS study is focused on the mechanism 


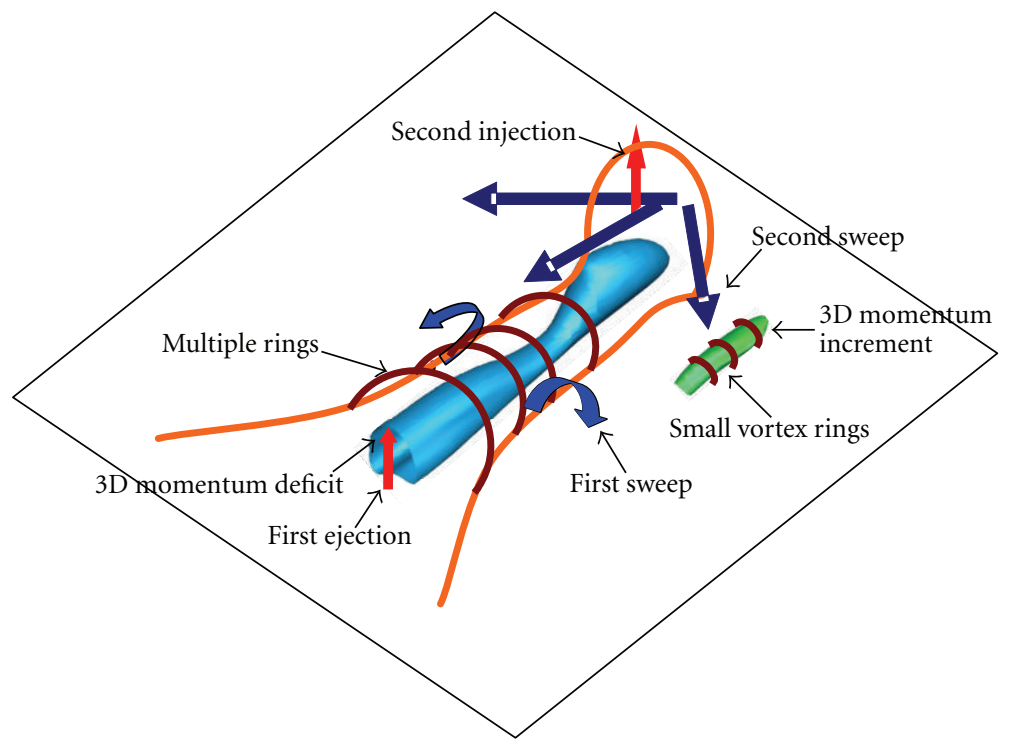

FIGURE 33: Sketch of mechanism of multiple rings formation and small vortices formation.

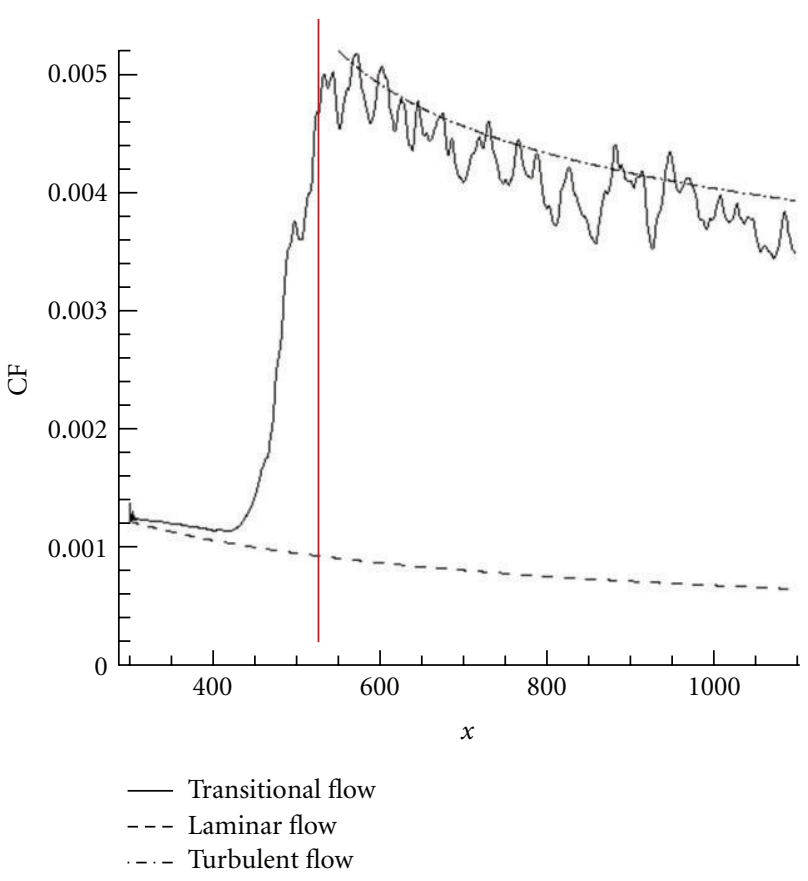

Figure 34: Maximum CF at $x=508.633$ (Laminar).

of randomization and the DNS computation only allows use of two periods in the spanwise direction, we consider that the flow is randomized when the symmetry is lost and period is changed from $\pi$ to $2 \pi$ (Figures $38(\mathrm{a})$ and $38(\mathrm{~b})$ ):

$$
\begin{aligned}
f(y)= & \sum_{k=0}^{n} a_{k} \cos (2 k y)+\sum_{k=1}^{n-1} b_{k} \sin (2 k y) \\
& +\sum_{k=0}^{n-1} c_{k} \cos (2 k y+y)+\sum_{k=0}^{n-1} d_{k} \sin (2 k y+y) .
\end{aligned}
$$

In real flow, there is no such a restriction of periodic boundary condition in the spanwise direction. A more detailed discussion about the mechanism of flow randomization will be given by another AIAA 2012 paper [36].

\section{Summary of Our DNS Findings}

4.1. Our New Observations. Since turbulence generation and sustenance are one of the top secret in nature, this research will bring significant impact on fundamental fluid mechanics as the classical theory and dominant concepts on late flow transition and turbulence structure are challenged. Table 2 gives a comparison of classical theory and currently dominant conclusions with the observation by our DNS.

\subsection{New Theory on Turbulence Formation and Sustenance by Liu}

4.2.1. Classical Theory on Turbulence. A turbulent flow is characterized by a hierarchy of scales through which the energy cascade takes place. According to Richardson, turbulence is random interactions of "eddies" as "big whorls have little whorls, little whorls have smaller whorls, that feed on their velocity, and so on to viscosity" (Figure 39 by [39]). On the other hand, vortex stretching is the core mechanism on which the turbulence energy cascade relies to establish the structure function. According to Richardson (1928) and Kolmogorov [6, 7], the radial length scale of the vortices decreases and the larger flow structures breakdown into smaller structures (Figure 40 by [40]). The process continues until the small-scale structures are small enough to the extent where their kinetic energy is overwhelmed by the fluid's molecular viscosity and dissipated into heat.

Richardson's energy cascade theory has several important points of view which can be summarized as follows: 


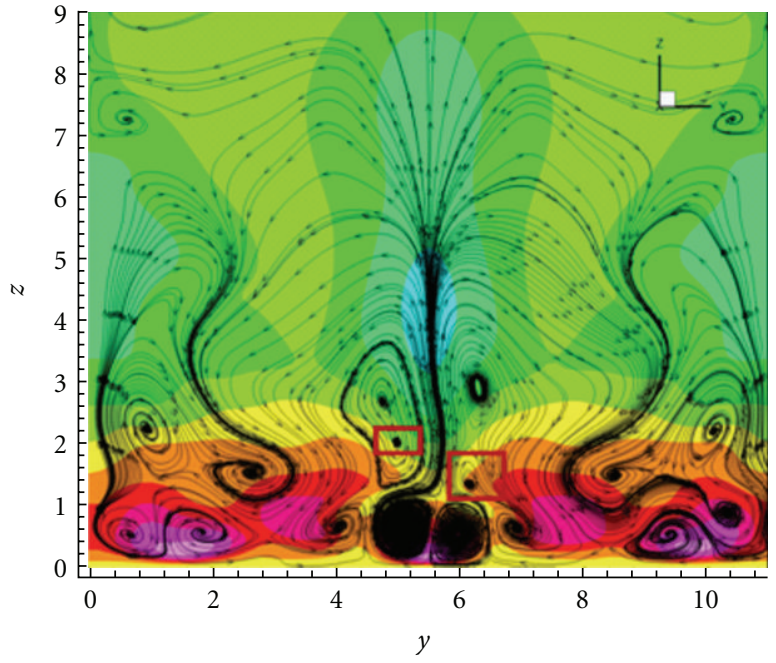

(a) Section view in $y$ - $z$ plane

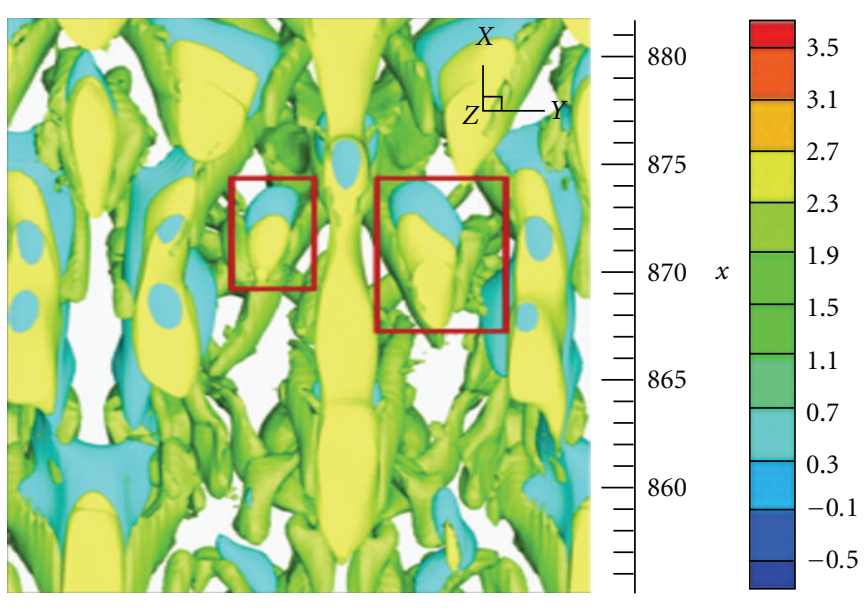

(b) Bottom view of positive spike

Figure 35: The flow lost symmetry in second level rings and bottom structure at $t=15.0 T$.

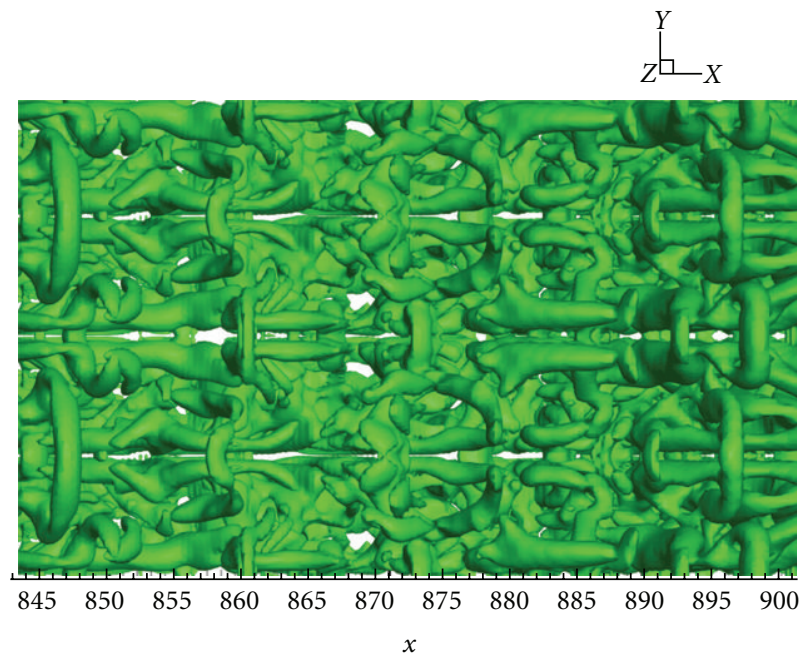

Figure 36: The top ring structure is still symmetric at $t=15.0 T$.

(1) stremwise vortex is stretching;

(2) vortex must break down to smaller structure due to the stretching;

(3) energy transfers from large vortex to smaller one through "vortex breakdown;"

(4) these smaller vortices continue the same process until the smallest one appears (Kolmgorov's length scale) in which viscosity is dominant;

(5) the micro-scale $\eta$ (Kolmogorov scale) is several order smaller than the macro-scale $L$ (main flowscale) and the middle length scales $r(\eta \ll r \ll L)$ are basically inertial.

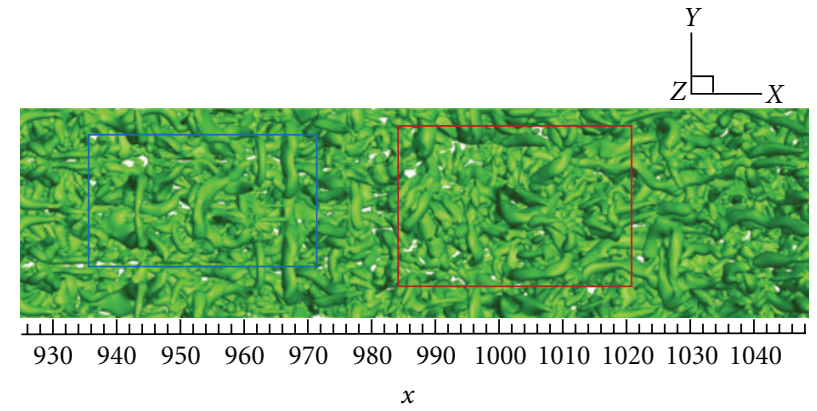

(a) Top ring structure lost symmetry (blue area is symmetric but red area is not)

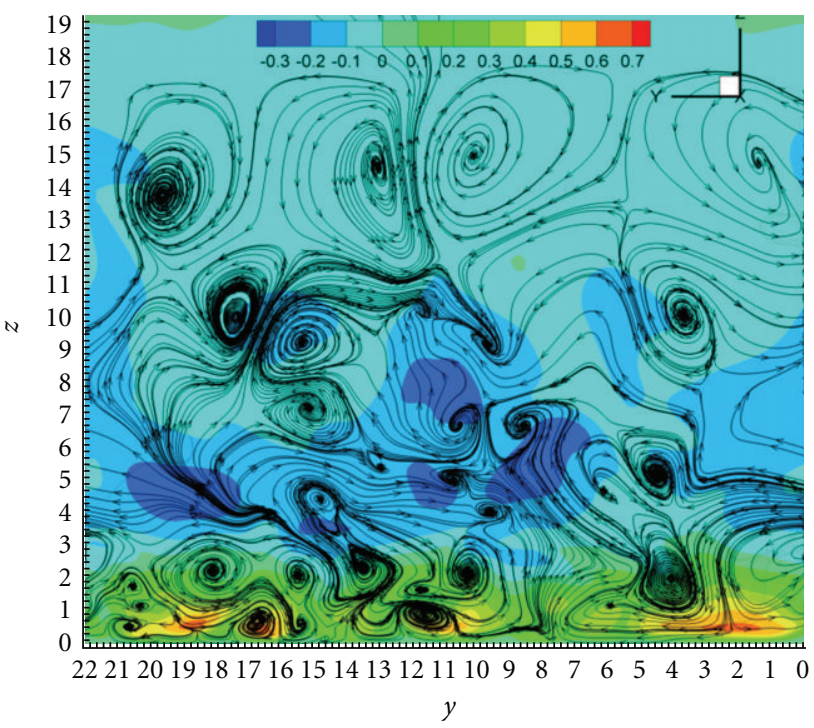

(b) Symmetry loss in the whole section of $y$ - $z$ plane

Figure 37: The whole flow field lost symmetry at $t=21.25 T$. 


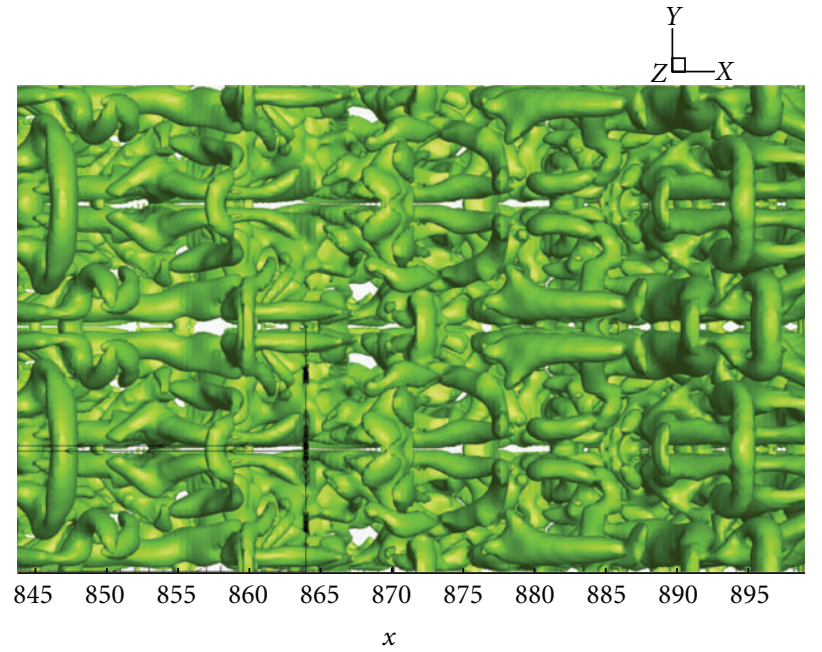

(a)

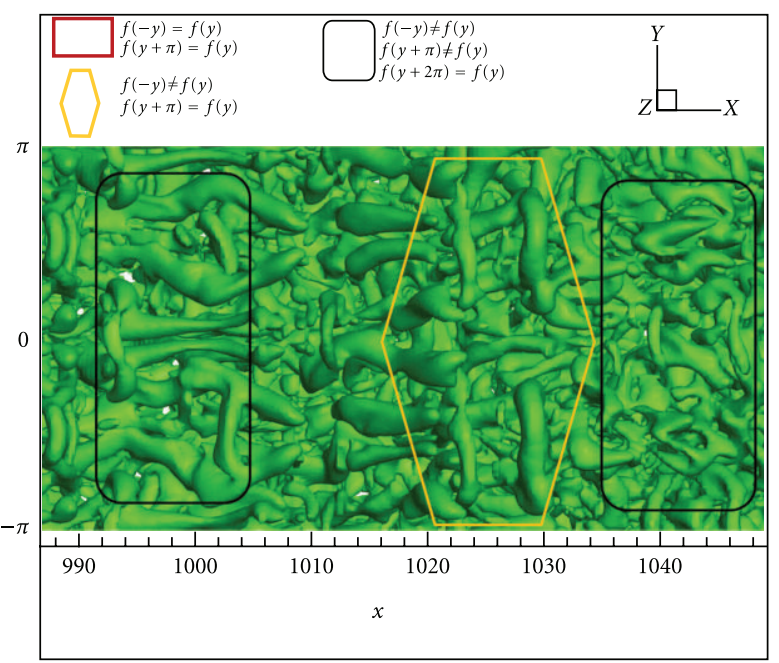

(c)

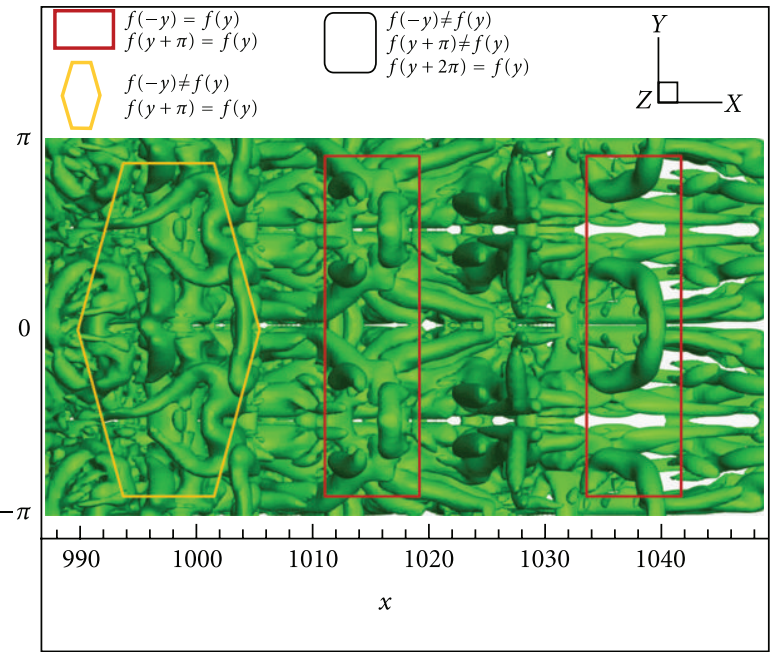

(b)

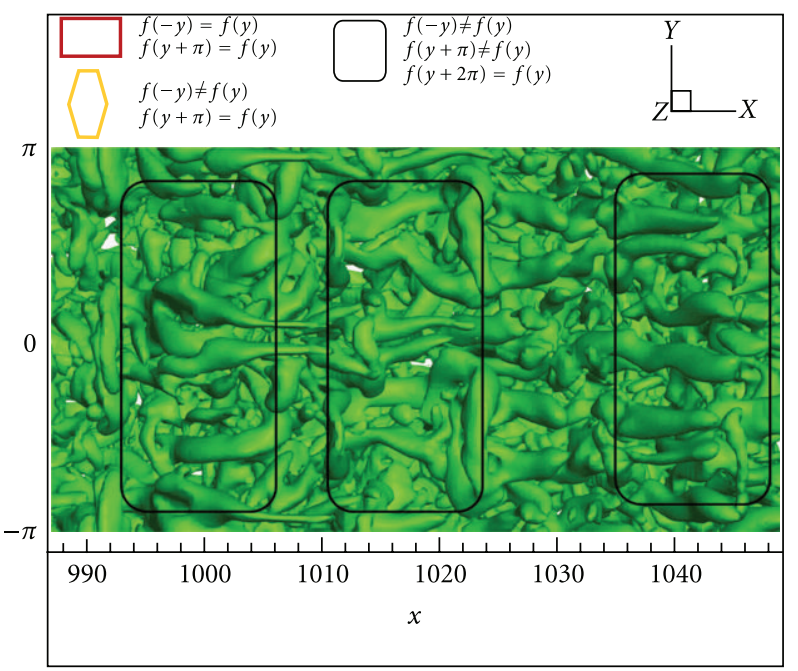

(d)

Figure 38: (a) Whole domain is symmetric and periodic $-a \times \cos 2 y$ (the period $=\pi$; spanwise domain is $-\pi<y<\pi$ ), (b) symmetric and asymmetric -redrectangular: periodic and symmetric at $y=-\pi / 2,0, \pi / 2$, that is, $f(-\pi / 2-y)=f(-\pi / 2+y), f(-y)=f(y), f(\pi / 2-y)=$ $f(\pi / 2+y)$; yellow diamond: periodic, $f(y+\pi)=f(y)$, period $=\pi$; but asymmetric $f(-y) \neq f(y)$; the spanwise domain is $-\pi<y<\pi$, (c) periodic but asymmetric-yellow diamond: periodic, period $=\pi$; black box: periodic but period $=2 \pi$; the spanwise domain is $-\pi<y<\pi$. (d) Periodic but asymmetric_all black boxes: periodic but asymmetric (period $=2 \pi$ ); the spanwise domain is $-\pi<y<\pi$.

4.2.2. New Observations and Analyses. On the other hand, our new observations can be described as follows:

(1) stremwise vortex is stretched but never breaks down;

(2) vortex has strong rotation which causes strong mixing;

(3) in the middle of two vortex legs, there is a lowspeed zone formed by ejection from boundary layer bottom to bring momentum deficit up to this area. This momentum deficit forms a long-shear layer;

(4) this shear layer in the middle of two legs is unstable and multiple rings with spanwise vorticity will form one by one to generate multiple rings near the inviscid region due to the shear layer instability especially near the narrowed vortex neck where rotation is very strong and the low speed momentum deficit is large. In this process, Helmholtz vorticity conservation must be satisfied;

(5) in the two sides of the vortex legs, high-speed zones are formed by sweeps which bring high energy (energy increment) from inviscid area to the near wall region. The energy increment will form shear layers near the wall;

(6) the momentum increment shear layers near the wall are unstable (shear layer instability) and will form multiple smaller ring-like vortices;

(7) these newly generated large and smaller vortices will continue a similar process to generate smaller ringlike vortices until the vortex size is too small and 

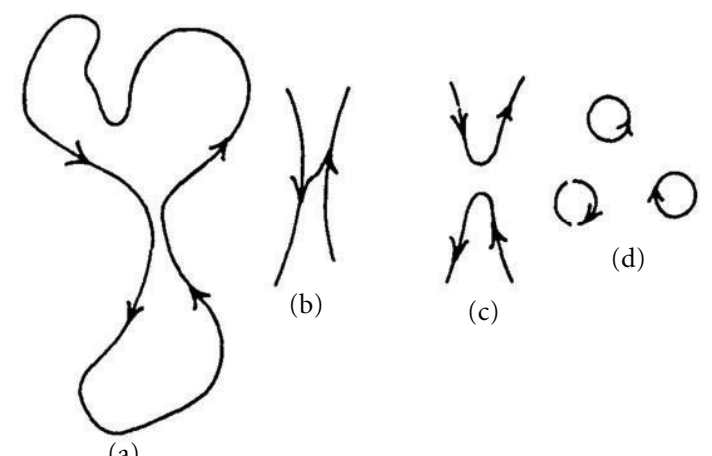

(c)

(a)

Figure 39: Sketch of vortex breakdown [39] (Tsubota et al, 2009). A vortex ring (a) can break up into smaller rigs if the transition between states (b) and (c) allowed when the separation of vortex lines becomes of atomic dimensions. The eventual small rings (d) may be identical to rotons.

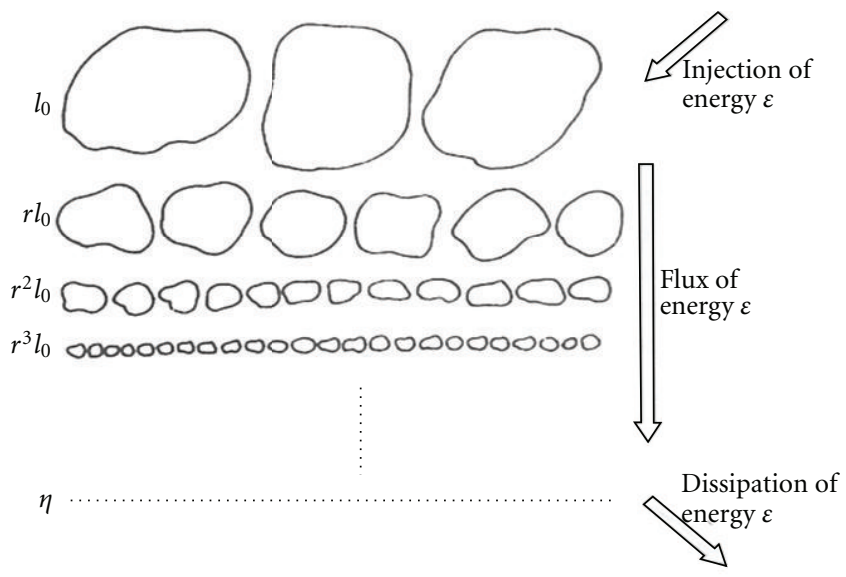

FIGURE 40: Sketch of Richardson cascade process [40].

the new shear layer becomes stable. The shear layer stability analysis should be further studied;

(8) energy is transferred from the large size vortices to small size vortices through multiple level sweeps. Dissipation is inevitable during the energy transfer;

(9) all smaller vortices are newly generated by shear layer instability, but not by original vortex "breakdown;"

(10) smallest length scales are all generated near the wall surface which must be related to the surface configuration and cannot be "isotropic;"

(11) surface drag is determined by small length scale generation, which must be closely related to turbulence modeling;

(12) "shear layer" control is the key issue for flow transition control, turbulent flow control, and drag reduction.

\subsection{Possible Impact on Fluid Mechanics}

4.3.1. Impact on Fundamental Fluid Mechanics. Since turbulence generation and sustenance are one of the top mysteries in nature, this research will bring significant impact on fundamental fluid mechanics as the classical theories on late flow transition and turbulence structure are challenged. LBLT classical theory, Richardson eddy cascade, Kolmogorov first and second hypotheses should be all revisited.

4.3.2. Impact on DNS and LES. As shown by the new DNS, it is not observed that "Kolmogorov scale" is the smallest turbulence scale but is found that the smallest length scale is determined by "shear layer instability". The estimate of energy containing scale, $\eta_{\min } / \eta_{\text {ref }}=(1 / \mathrm{Re})^{3 / 4}$, is too conservative. For example, an engineering hydrodynamics problem has commonly a Reynolds number, $\operatorname{Re}=10^{8}$. Considering 3D in space and $1 \mathrm{D}$ in time, the requirement for computation would be $Q=\operatorname{Re}^{4 \times 3 / 4}=\left(10^{8}\right)^{3}=10^{24}$. This is apparently impossible for any currently available computer or any near future computers. However, researchers in US National Research Labs and many others around the world do "implicit LES" which is really "coarse grid DNS". They do DNS, but are afraid to call DNS but "ILES". The reason is that they are afraid of "Kolmogorov scale." Since we could not find that energy is transformed from large vortices to small vortices by "vortex breakdown", "Kolmogorov scale" would lose solid scientific foundation and must be revisited. This will provide guidance to DNS which is supposed to resolve all energy-contained small length scales and LES which is expected to resolve the large eddies and model the subgird scales.

4.3.3. Impact on Turbulence Modeling. The eddy viscosity turbulence model is mainly based on "Boussinesq Assumption" (1872) that the nonlinear perturbation terms can be modeled by resolved mean scales with new turbulent coefficients of viscosity. However, most turbulence models are still empirical and case-related. The right turbulence models can be developed with deep understanding of turbulence generation and sustenance. As shown by our DNS, the perturbation and small length scales are not isotropic but configuration related. Yes, Prandtl may be right that the turbulence can be described by "mixing lengths" which represent the length of different size vortices. Although Prandtl's "mixing length" model is an old concept [42], turbulence is really generated by mixing of variety of different size vortices and the "mixing length" concept coincides with physics. The serious question is how to find the right size of "mixing length". We should develop a new "mixing length" model based on DNS data and "shear layer stability" analysis.

4.3.4. Impact on Flow Control of Flow Transition and Turbulence. Flow transition and turbulence control should be based on right understanding on flow transition and turbulence structure. Based on our observation, it is proposed to cut the energy transfer channels for high shear layer generation to stop or delay turbulence generation. It is also found that the "shear layer instability" is the "mother of turbulence." Apparently, control of shear layer formation and structure is the key issue to the control of the 
TABLE 2: Comparison of classical theory and our DNS observation.

\begin{tabular}{|c|c|c|}
\hline Topic & Classical or existing theory & Observation of our DNS \\
\hline Turbulence generation & By "vortex breakdown" & $\begin{array}{l}\text { Not by "vortex breakdown" but by shear layer } \\
\text { instability }\end{array}$ \\
\hline First-ring generation & Self-induced, deformed, inclined, and pinched-off & $\begin{array}{l}\text { By counter-rotated vortices interaction, circular, } \\
\text { perpendicular, no pinch-off }\end{array}$ \\
\hline Multiple-ring structure & $\begin{array}{l}\text { "Crow theory" or breakdown and then } \\
\text { reconnected }\end{array}$ & $\begin{array}{l}\text { No breakdown, not "Crow theory" but momentum } \\
\text { deficit caused by ejection, vorticity conservation }\end{array}$ \\
\hline Multiple-level high shears & No report & By multiple-level sweeps and ejections \\
\hline $\begin{array}{l}\text { Energy transfer channel, } \\
\text { turbulence sustenance }\end{array}$ & $\begin{array}{l}\text { Energy transfers from larger vortices to smaller } \\
\text { one through "vortex breakdown" without } \\
\text { dissipation until viscosity }\end{array}$ & $\begin{array}{l}\text { From inviscid flow down to bottom by multilevel } \\
\text { sweeps }\end{array}$ \\
\hline U-shaped vortex & $\begin{array}{l}\text { Head wave, secondary vortex, by second sweep, } \\
\text { newly formed, breakdown }\end{array}$ & $\begin{array}{l}\text { Not head wave, tertiary vortex, by secondary vortex, } \\
\text { existing from beginning, never breakdown }\end{array}$ \\
\hline Randomization & $\begin{array}{l}\text { Background noise, starting from the top ring and } \\
\text { then going down to the bottom }\end{array}$ & $\begin{array}{l}\text { Internal property, starting from second level rings in } \\
\text { the middle, affects bottom and then up to affect top } \\
\text { rings. Loss of symmetry maybe caused by C-K shift }\end{array}$ \\
\hline Coefficients of friction & $\begin{array}{l}\text { Turbulent flow has large friction due to strong } \\
\text { boundary layer mixing }\end{array}$ & $\begin{array}{l}\text { Depending only on velocity profile changes in laminar } \\
\text { sublayer, no matter turbulent or laminar }\end{array}$ \\
\hline Richardson eddy cascade & Classical theory & Not observed \\
\hline Vortex breakdown & Classical theory & Not observed \\
\hline Kolmogorov scale & Classical theory & $\begin{array}{l}\text { Smallest length scale should be determined by } \\
\text { minimum shear layer instability }\end{array}$ \\
\hline
\end{tabular}

transitional flow and fully developed turbulent flow. It is also an important way for drag production of turbulent flow, for example, why oscillating wall and rough wall with riblets can reduce the surface drag.

\section{Conclusions}

In our recent DNS work [20-32, 36, 37] we have made a number of new findings on late flow transition in a boundary layer including the mechanism of large vortex structure formation, small-length scale generation and flow randomization (loss of symmetry).

New theory on flow transition and turbulence generation and sustenance has been given by Liu. Instead of four steps, that is, receptivity, linear instability, non-linear growth, vortex breakdown to turbulence, described by classical theory, Liu proposed a new theory with five steps, that is, receptivity, linear instability, large vortex formation, small length scale generation, loss of symmetry and randomization to turbulence. For turbulence generation and sustenance, the classical theory, described with Richardson's energy cascade and Kolmogorov length scale, is not observed by our DNS. Liu proposed a new theory on turbulence generation that all small length scales are generated by "shear layer instability" through multiple level ejections and sweeps and consequent multiple level positive and negative spikes, but not by "vortex breakdown" which can never happen. We believe "shear layer instability" is the "mother of turbulence." Flow randomization is caused by the internal property of instability of the structure of multiple vortex-ring-spot overlapping.

\section{Nomenclature}

$\begin{array}{ll}M_{\infty}: & \text { Mach number } \\ \text { Re: } & \text { Reynolds number } \\ \delta_{\text {in }}: & \text { Inflow displacement thickness } \\ T_{w}: & \text { Wall temperature } \\ T_{\infty}: & \text { Free stream temperature } \\ L z_{\text {in }}: & \text { Height at inflow boundary } \\ L z_{\text {out }}: & \text { Height at outflow boundary } \\ L x: & \text { Length of computational domain along } \\ L y: & \text { Length of computational domain along } y \\ & \text { direction } \\ x_{\text {in }}: & \text { Distance between leading edge of flat plate } \\ & \text { and upstream boundary of computational } \\ A_{2 d}: & \text { domain } \\ A_{3 d}: & \text { Amplitude of } 2 \mathrm{D} \text { inlet disturbance } \\ \omega: & \text { Frequency of inlet disturbance } \\ \alpha_{2 d}, \alpha_{3 d}: & \text { Two and three dimensional streamwise } \\ \beta: & \text { wave number of inlet disturbance } \\ \beta_{0}: & \text { Spanwise wave number of inlet } \\ \gamma_{0}: & \text { disturbance } \\ \mu_{\infty}: & \text { Ratio of specific heats } \\ & \text { Viscosity. }\end{array}$

\section{Acknowledgments}

This work was supported by AFOSR Grant FA9550-08-10201 supervised by Dr. John Schmisseur and the Department of Mathematics at University of Texas at Arlington. 
The authors are grateful to Texas Advanced Computing Center (TACC) for providing computation hours. This work is accomplished by using Code DNSUTA, which was released by Dr. Chaoqun Liu at University of Texas at Arlington in 2009.

\section{References}

[1] A. Marshak, 3D Radiative Transfer in Cloudy Atmospheres, Springer, New York, NY, USA, 2005.

[2] T. Mullin, "Turbulent times for fluids," New Scientist, vol. 124, no. 1690, pp. 52-55, 1989.

[3] P. A. Davidson, Turbulence: An Introduction for Scientists and Engineers, Oxford University Press, 2004.

[4] V. I. Borodulin, V. R. Gaponenko, Y. S. Kachanov et al., "Latestage transitional boundary-layer structures. Direct numerical simulation and experiment," Theoretical and Computational Fluid Dynamics, vol. 15, no. 5, pp. 317-337, 2002.

[5] C. Lee and R. Li, "Dominant structure for turbulent production in a transitional boundary layer," Journal of Turbulence, vol. 8, pp. 1-34, 2007, N55.

[6] A. N. Kolmogorov, "The local structure of turbulence in incompressible viscous fluid for very large Reynolds numbers," in Proceedings of the USSR Academy of Sciences, vol. 30, pp. 299-303, 1941.

[7] A. N. Kolmogorov, "The local structure of turbulence in incompressible viscous fluid for very large Reynolds numbers," Proceedings of the Royal Society of London A, vol. 434, pp. 9-13, 1991.

[8] U. Frisch, Turbulence: The Legacy of A. N. Kolmogorov, Cambridge University Press, New York, NY, USA, 1995.

[9] B. Singer and R. Joslin, "Metamorphosis of a hairpin vortex into a young turbulent spot," Physics of Fluids, vol. 6, no. 11, pp. 3724-3736, 1994.

[10] T. Herbert, "Secondary Instability of Boundary Layer," Annual Review of Fluid Mechanics, vol. 20, pp. 487-526, 1988.

[11] Y. S. Kachanov, "Physical mechanisms of laminar-boundarylayer transition," Annual Review of Fluid Mechanics, vol. 26, no. 1, pp. 411-482, 1994.

[12] L. Kleiser and T. A. Zang, "Numerical simulation of transition in wall-bounded shear flows," Annual Review of Fluid Mechanics, vol. 23, no. 1, pp. 495-537, 1991.

[13] D. Sandham and L. Kleiser, "The late stages of transition in channel flow," Journal of Fluid Mechanics, vol. 245, pp. 319348, 1992.

[14] U. Rist and Y. S. Kachanov, "Numerical and experimental investigation of the K-regime of boundary-layer transition," in Laminar-Turbulent Transition, R. Kobayashi, Ed., pp. 405-412, Springer, Berlin, Germany, 1995.

[15] S. Bake, D. G. W. Meyer, and U. Rist, "Turbulence mechanism in Klebanoff transition: a quantitative comparison of experiment and direct numerical simulation," Journal of Fluid Mechanics, vol. 459, pp. 217-243, 2002.

[16] Y. S. Kachanov, "On a universal mechanism of turbulence production in wall shear flows," in Notes on Numerical Fluid Mechanics and Multidisciplinary Design, vol. 86 of Recent Results in Laminar-Turbulent Transition, pp. 1-12, Springer, Berlin, Germany, 2003.

[17] R. J. Adrian, "Hairpin vortex organization in wall turbulencea," Physics of Fluids, vol. 19, no. 4, Article ID 041301, 2007.
[18] X. Wu and P. Moin, "Direct numerical simulation of turbulence in a nominally zero-pressure-gradient flat-plate boundary layer," Journal of Fluid Mechanics, vol. 630, pp. 541, 2009.

[19] H. Guo, V. I. Borodulin, Y. S. Kachanov et al., "Nature of sweep and ejection events in transitional and turbulent boundary layers," Journal of Turbulence, vol. 11, pp. 1-9, 2010.

[20] L. Chen, X. Liu, M. Oliveira, D. Tang, and C. Liu, "Vortical structure, sweep and ejection events in transitional boundary layer," Science China Physics, Mechanics and Astronomy, vol. 39, no. 10, pp. 1520-1526, 2009.

[21] L. Chen, X. Liu, M. Oliveira, and C. Liu, "DNS for ring-like vortices formation and roles in positive spikes formation," AIAA Paper 2010-1471, Orlando, Fla, USA, 2010.

[22] L. Chen, D. Tang, P. Lu, and C. Liu, "Evolution of the vortex structures and turbulent spots at the late-stage of transitional boundary layers," Science China, vol. 54, no. 5, pp. 986-990, 2011.

[23] L. Chen and C. Liu, "Numerical study on mechanisms of second sweep and positive spikes in transitional flow on a flat plate," Computers and Fluids, vol. 40, no. 1, pp. 28-41, 2011.

[24] X. Liu, L. Chen, M. Oliveira, D. Tang, and C. Liu, "DNS for late stage structure of flow transition on a flat-plate boundary layer," AIAA Paper 2010-1470, Orlando, Fla, USA, 2010.

[25] C. Liu and L. Chen, "Study of mechanism of ring-like vortex formation in late flow transition," AIAA Paper 2010-1456, Orlando, Fla, USA, 2010.

[26] X. Liu, Z. Chen, and C. Liu, "Late-stage vortical structures and eddy motions in transitional boundary layer status," Chinese Physics Letters, vol. 27, no. 2, 2010.

[27] C. Liu, L. Chen, and P. Lu, "New findings by high-order DNS for late flow transition in a boundary layer," Modelling and Simulation in Engineering, vol. 2011, Article ID 721487, 2011.

[28] C. Liu and L. Chen, "Parallel DNS for vortex structure of late stages of flow transition," Computers and Fluids, vol. 45, no. 1, pp. 129-137, 2011.

[29] C. Liu, "Numerical and theoretical study on "Vortex Breakdown"', International Journal of Computer Mathematics, vol. 88, no. 17, pp. 3702-3708, 2011.

[30] C. Liu, L. Chen, P. Lu, and P. Liu, "Study on multiple ringlike vortex formation and small vortex generation in late flow transition on a flat plate," 2011, Theoretical and Numerical Fluid Dynamics. In press.

[31] P. Lu and C. Liu, "Numerical study of mechanism of u-shaped vortex formation," Journal of Computers and Fluids, pp. 36-47, 2012, AIAA Paper 2011-0286.

[32] P. Lu and C. Liu, "DNS study on mechanism of small length scale generation in late boundary layer transition," Journal of Physica D, vol. 241, no. 2012, pp. 11-24, 2011.

[33] J. Jeong and F. Hussain, "On the identification of a vortex," Journal of Fluid Mechanics, vol. 285, pp. 69-94, 1995.

[34] L. Jiang, C. L. Chang, M. Choudhari, and C. Liu, "Crossvalidation of DNS and PSE results for instability-wave propagation," in Proceedings of the 16th American Institute of Aeronautics and Astronautics Computational Fluid Dynamics Conference (AIAA '03), Orlando, Fla, USA, June 2003, AIAA Paper \#2003-3555.

[35] F. Ducros, P. Comte, and M. Lesieur, "Large-eddy simulation of transition to turbulence in a boundary layer developing spatially over a flat plate," Journal of Fluid Mechanics, vol. 326, pp. 1-36, 1996.

[36] P. Lu, M. Thampa, and C. Liu, "Numerical study on randomization in late boundary layer transition," in American Institute 
of Aeronautics and Astronautics Scientific Meeting (AIAA '12), Nashville, Tenn, USA, January 2012, AIAA Paper 2012-0747.

[37] P. Lu and C. Liu, "Numerical study on mechanism of multiple ring formation," AIAA Paper 2012-0747, Nashville, Tenn, USA, 2012.

[38] P. Moin, A. Leonard, and J. Kim, "Evolution of a curved vortex filament into a vortex ring," Physics of Fluids, vol. 29, no. 4, pp. 955-963, 1986.

[39] R. F. Feynman, "Application of quantum mechanics to liquid helium," in Progress in Low Temperature Physics, C. J. Gorter, Ed., vol. 1, chapter 2, North Holland, Amsterdam, The Netherlands, 1955.

[40] U. Frisch, P. L. Sulem, and M. Nelkin, "A simple dynamical model of intermittent fully developed turbulence," Journal of Fluid Mechanics, vol. 87, no. 4, pp. 719-736, 1978.

[41] D. G. W. Meyer, U. Rist, and M. J. Kloker, "Investigation of the flow randomization process in a transitional boundary layer," in High Performance Computing in Science and Engineering '03, E. Krause and W. Jäger, Eds., pp. 239-253, Springer, New York, NY, USA, 2003.

[42] L. Prandtl, "Bericht uber Untersuchungen zur ausgebildeten Turbulenz," Zeitschrift für Angewandte Mathematik und Mechanik, Band, vol. 5, pp. 136-139, 1925. 

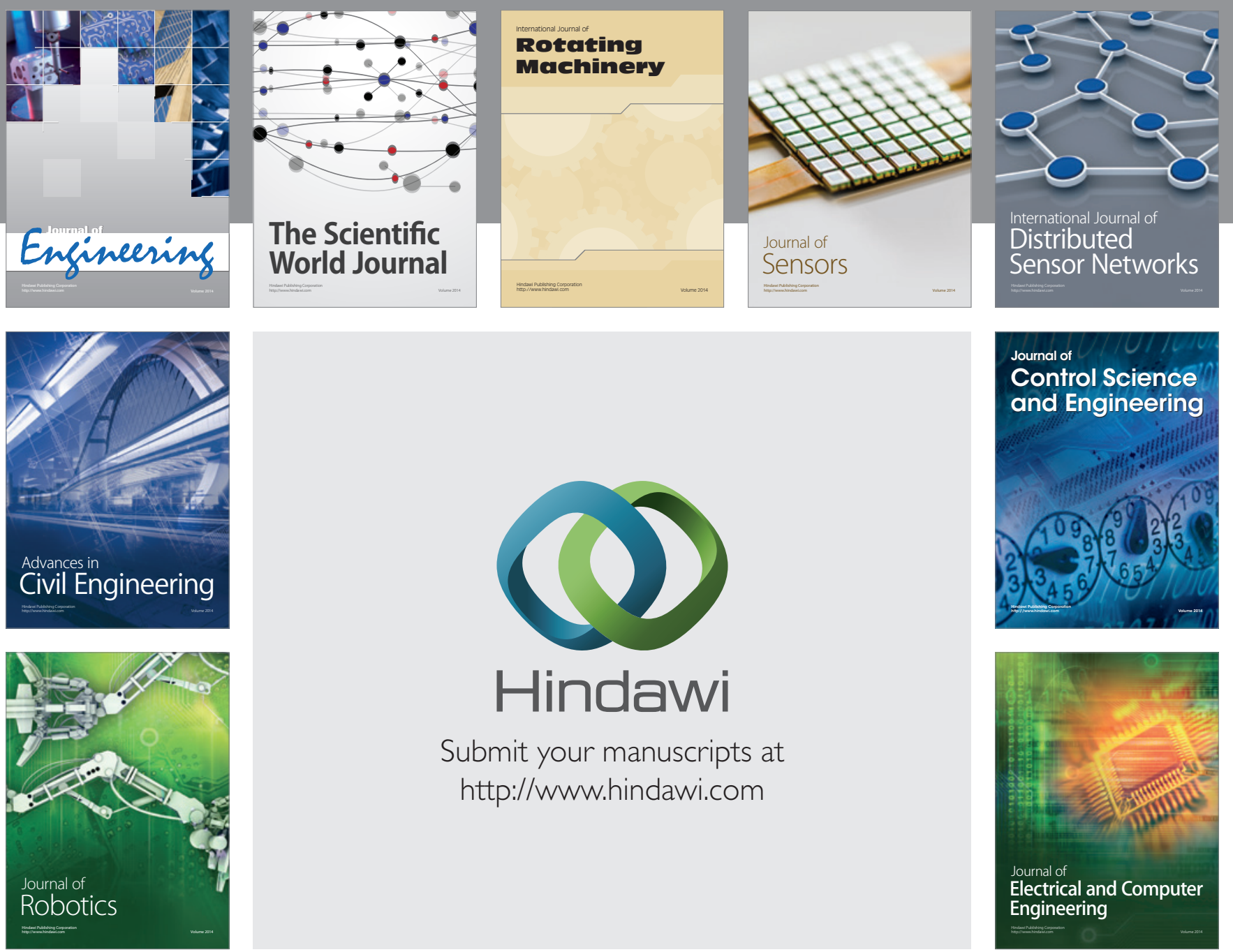

Submit your manuscripts at

http://www.hindawi.com
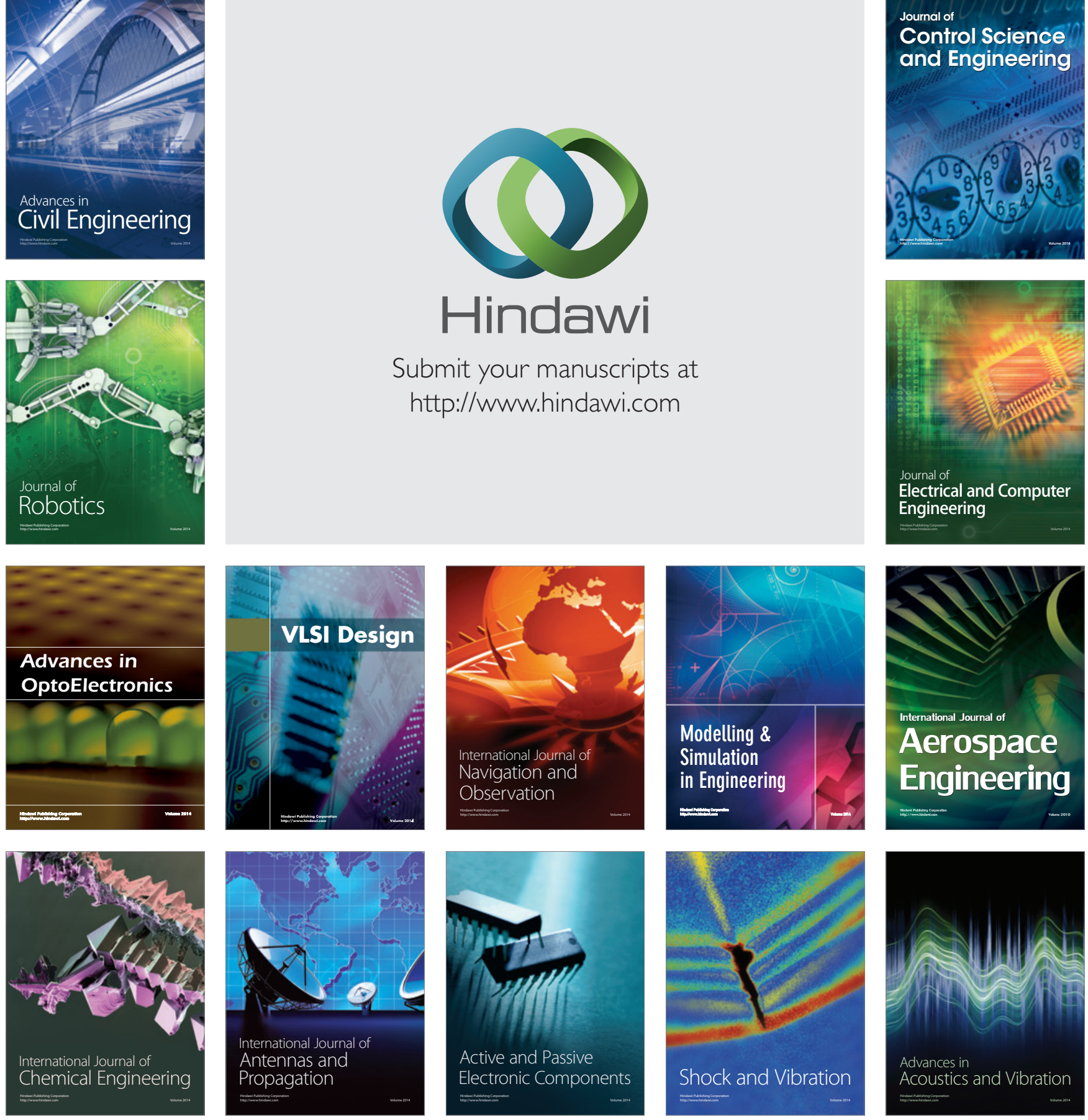